\title{
La gestión por competencias para el desarrollo organizacional en la Universidad Nacional Mayor de San Marcos
}

Lucía Fernanda Palmira Castillo Arias ${ }^{1}$

ReCibido: 17/06/2019 Aceptado: 25/02/2021 Publicado: 26/07/2021

\begin{abstract}
RESUMEN
El presente estudio pretende contribuir con la mejora de la administración de recursos humanos mediante un modelo de gestión enfocado en competencias para los cargos administrativos de la administración central que conduzca al desarrollo organizacional en la Universidad Nacional Mayor de San Marcos. Esto porque, con frecuencia, las personas que ejercen cargos administrativos no cuentan con las habilidades requeridas para gestionar, sino solo con conocimientos técnicos, lo cual no es suficiente para alcanzar los objetivos establecidos en el plan estratégico de la universidad. Se revisaron distintas fuentes bibliográficas, estudios relativos al tema, así como la metodología de trabajo establecida por SERVIR. El estudio puso en evidencia que no se han establecido medidas específicas para los perfiles de los cargos directivos en la universidad, por lo que es necesario que la oficina de recursos humanos implemente un modelo de gestión por competencias e inste a cumplir con los lineamientos establecidos. La introducción de una nueva propuesta basada en competencias en la universidad invita a rediseñar los perfiles de trabajo en cuanto a funciones de desempeño.
\end{abstract}

Palabras clave: talento humano; competencias; gestión; universidad.

\section{INTRODUCCIÓN}

En la actualidad, las universidades públicas y privadas están utilizando nuevos modelos de dirección por competencias en el área de recursos humanos con la finalidad de incorporar tácticas competitivas que hagan frente a los cambios globales y satisfagan las necesidades del intelecto humano; sobre esta base, el presente trabajo académico tiene como objetivo general mejorar la dirección de recursos humanos bajo el contexto de competencias que permita el desarrollo organizacional en la universidad. Por lo cual es necesario revisar diversas fuentes bibliográficas, estudios sobre el tema y los lineamientos de la Autoridad Nacional del Servicio Civil (SERVIR) como ente regidor de los servidores públicos.

Esta investigación propone un estudio sobre la administración de recursos humanos basado en competencias en las universidades, públicas o privadas, mediante un modelo que detalla los perfiles de desempeño por competencias para los cargos administrativos y que pueda ser utilizado en futuras investigaciones. Propone además establecer una gestión por competencias en el ambiente institucional de la universidad, que contribuya con la elaboración de los perfiles con el fin de incrementar la productividad administrativa.

A lo largo de los años, la universidad peruana ha atravesado diferentes contextos de cambio en su organización, en donde se ha observado que el personal que toma decisiones reacciona ante los distintos escenarios o coyunturas cuando estos se presentan, en lugar de anticiparse a ellos, lo que provoca alteraciones y/o intervenciones políticas que crean un desbalance en el clima organizacional. Además, las personas que ocupan cargos administrativos, con frecuencia, carecen de las habilidades necesarias para gestionar, pero son contratados por sus conocimientos de carrera y/o técnicos, los cuales no son suficientes para liderar equipos de trabajo y tomar decisiones. Dentro de la universidad, la manera en que los responsables de la gerencia

Licenciada en Administración de Empresas por la Universidad Inca Garcilaso de la Vega Actualmente, es jefe de marketing del Centro de Informática de la Universidad Nacional Mayor de San Marcos. (Lima, Perú)

ORCID: https://orcid.org/0000-0001-8632-6906

Autor de correspondencia: 1castilloa1@unmsm.edu.pe 
realizan sus funciones se relaciona directamente con el desarrollo organizacional (DO), de ahí la importancia de que los integrantes estén satisfechos con el desarrollo de sus funciones para que así, en equipo, alcancen el éxito y los objetivos institucionales. La función principal de los cargos administrativos dentro de la universidad pública es el manejo eficiente de los recursos propios u ordinarios.

\section{MARCO TEÓRICO}

Delgado (2002) afirma que una administración adecuada consiste en usar eficaz y eficientemente los recursos de la universidad, es decir, a todos los integrantes de una comunidad universitaria, con el fin de lograr los objetivos. Aquellos que dirigen la universidad deben tener una idea de quiénes integran esta comunidad. Una nueva forma de gestionar una universidad debe apostar por un equipo de gestión que trabaje realizando sus funciones con responsabilidad, agilidad y eficacia.

En consecuencia, para una adecuada gerencia, las oficinas de recursos humanos deben considerar utilizar estándares de competencias, ya que al contar con personal idóneo será posible cumplir con los objetivos institucionales como parte del desarrollo organizacional; asimismo, mediante la consecución de objetivos se identifican las destrezas que un individuo tiene para realizar las funciones del puesto del trabajo.

De las diferentes fuentes bibliográficas estudiadas, se observó que la gestión por competencias es un prototipo de dirección que permite alinear al capital humano con la esencia de la organización, de modo que los trabajadores se encaminen profesionalmente, lo que ayuda con el cumplimiento del plan estratégico.

Al respecto, son varios los estudios que han tratado el tema dentro de la dirección de recursos humanos en universidades. Uno de estos postula la necesidad de implementar un modelo de gestión basado en competencias en los perfiles administrativos de una universidad, puesto que a) trabajar con un enfoque de competencias ayuda al aprendizaje continuo, a incorporar programas de capacitación y a adecuarse a la vanguardia tecnológica; b) especifica el perfil del personal idóneo y así se conoce lo que se espera del personal administrativo-docente y cómo las competencias ayudan eficientemente al logro de los objetivos; c) la selección del personal ya no debe basarse únicamente en grados académicos, sino también en las capacidades que debe poseer el personal apto para el perfil del puesto; y d) permite modernizar el perfil adecuado con el fin de relacionar los planes de desarrollo con las necesidades de cada área (Rodríguez, 2015).

En las organizaciones, la gestión del talento humano consiste en valorar a los colaboradores, mejorar su productividad y conservarlos, dado que son elementos necesarios para el manejo eficiente de la organización; de ahí que la administración del capital humano sea un área perceptible en la cultura organizacional, toda vez que los individuos pasan la mayor parte de sus vidas laborando, y de ellos dependen las organizaciones para operar y alcanzar sus objetivos (Chiavenato, 2002).

En "El capital humano", Becker (1964) se dedicó al estudio de las sociedades de la información, y determinó que el capital más valioso para una organización es el ser humano, puesto que posee nociones y destrezas que demuestra en sus hábitos laborales. Entonces, el autor conceptualiza al talento humano como factor principal para el rendimiento de las economías actuales en vista de que su productividad se sustenta en sus conocimientos.

Alles (2008) señala que los modelos actuales de gestión empresarial promueven el desarrollo de los colaboradores en función de los planes estratégicos de la organización, así pues, es posible que todos los integrantes de la organización se involucren con la estrategia organizacional y sean partícipes de su ejecución, lo que conduce al logro de los objetivos trazados.

McClelland (1973), en "Testing for Competence rather than for Intelligence", define el término "competencia" como el principal rasgo del ser humano, al tratarse de la fuente de su productividad para el desempeño de sus funciones en el trabajo.

Por su parte Spencer y Spencer (1993) definen a las competencias como atributos principales del ser humano, los cuales se muestran en su comportamiento o manera de pensar.

El concepto englobado de gestión por competencias señala que es un medio perenne de integración donde la organización incorpora las necesidades y los anhelos de los trabajadores con el propósito de ayudarlos y respaldarlos a fin de continúen involucrándose con los objetivos institucionales. La gerencia del talento humano para Fernández (2006) consiste en el desarrollo de nuevas propuestas para facilitar la gestión de los trabajadores y su adaptación a las necesidades de la empresa.

La gestión por competencias no solo representa el futuro, sino también la necesidad actual de una 
nueva generación laboral en las organizaciones. Es la técnica que reconocerá las principales capacidades que una persona necesita para cumplir con el perfil del puesto, lo que permitirá conservar el rendimiento elevado o superior e incorporar al individuo idóneo dentro de la organización, lo que a su vez generará ventajas competitivas para el desarrollo organizacional.

Son varios los autores que han dado aportes acerca del DO, el cual se entiende como una táctica administrativa que hace posible el cumplimiento del plan estratégico a través del buen uso de las herramientas de gestión y las de recursos humanos. De la Cruz (1999) ve el desarrollo organizacional como un procedimiento en donde se toman en cuenta todos los elementos relacionados al cambio para desarrollar un nuevo modelo de organización, enfocándose exclusivamente en el factor humano.

El DO en la organización debe orientarse en establecer la misión, visión, valores institucionales, tácticas y la práctica de estas, con el fin de que todos los trabajadores puedan adaptarse a los cambios continuos.

Sánchez (2009) indica que un apropiado proceso administrativo, junto con la evaluación de rendimiento del personal, logra el éxito de la estrategia competitiva, sumando a la premisa: el capital humano y su aspiración al logro de los objetivos deja en segundo plano a las finanzas y la tecnología.

Por otro lado, el escritor clásico del desarrollo organizacional, Bennis (1997, como se citó en Sánchez, 2009), explica que se trata de la respuesta al cambio, es una compleja estrategia educativa que busca cambiar las actitudes administrativas en la organización y estructurarla, de manera que pueda adecuarse a los cambios globales en tendencias organizacionales, tales como nuevas tecnologías, mercados y retos empresariales.

Chiavenato (2006) conceptualiza al DO como un procedimiento que se desarrolla a largo plazo, el cual es liderado por la gerencia general de la organización, que permite que los procesos de la organización sean más efectivos y que contribuye al desarrollo del personal.

Partiendo de las definiciones del DO, el vínculo entre el desarrollo organizacional y la gestión universitaria puede reformarse en procesos de la organización, con sus respectivas características teóricas, metodológicas y operacionales, que permitan el cumplimiento de sus objetivos como institución.
Espinoza (2000) señala que:

Hoy en día, la premisa "capacidad gerencial demostrada" en la gerencia universitaria no es un elemento valorativo para componer la estructura gerencial de la universidad, queriendo significar que el personal que ocupa cargos de gestión muchas veces están ahí por cuestiones políticas y/o de poder político. (pág. 87)

Según Fernández (2008):

La Universidad es un centro generador de conocimiento, y por ello su rol y efecto en el desarrollo de las sociedades y de los países es fundamental. Sin embargo, principalmente en el caso de la universidad pública, existen varios factores que impiden su mayor competitividad y liderazgo; y por lo tanto su aporte en el desarrollo del país. Un factor podría ser la forma de gobierno o gestión universitaria; otra sería su cultura y/o medioambiente organizacional. La Universidad pública peruana presenta diversos problemas que suceden en un entorno interno y externo adversos. Internamente, puede estar la falta de una gestión universitaria que defina e implemente estrategias para la competitividad y calidad; y externamente, el rol del Estado, por su falta de intervención y definición de una política educativa como estrategia de desarrollo y bienestar. (pág. 3)

La Universidad Nacional Mayor de San Marcos (UNMSM) es una entidad pedagógica universitaria encaminada a la perfección y liderato. En ese sentido, aquellos que participan en su gestión deben cumplir con los objetivos corporativos y tener visión ante los cambios invariables que la gestión universitaria exige. Se requiere de personal comprometido, identificado y con altos niveles de competencia para conseguir un desarrollo organizacional estable y continuo; asimismo, es necesario proporcionar calidad de servicio e impulsar la concreción de los objetivos estratégicos. Es por ello que se debe gestionar el perfeccionamiento de conocimientos a través de capacitaciones para los cargos administrativos.

El Reglamento de Organización y Funciones (ROF) aprobado con R. R. Nº1206-R-11 (2011) es el documento oficial que formaliza a la UNMSM como institución educativa, detalla su naturaleza y competencia, detalla las ocupaciones generales y específicas de cada uno de sus órganos y sus unidades orgánicas. 
El personal administrativo de las instituciones educativas públicas del Perú trabaja con base en los lineamientos de SERVIR, cumpliendo con regímenes de capacitación y desarrollo continuo de los servidores públicos. La presente investigación se apoyó en los lineamientos del "Manual de Perfiles de Puestos (MPP)", aprobado con la Directiva $\mathrm{N}^{\circ}$ 001-2016-SERVIR/GDSRH (2016).

\section{METODOLOGÍA}

El estudio realizado es de tipo mixto, cualitativo por la recaudación de información que expresa cualidad, y cuantitativo porque se recopiló información utilizando indicadores y se realizó el análisis de datos con programas estadísticos. Es de carácter descriptivo-correlacional, pues su finalidad es medir la relación existente entre la gestión por competencias y el desarrollo organizacional en la universidad; y de diseño no experimental. Asimismo, se analizaron las opiniones de los encuestados a través de sus contestaciones y observaciones; de este modo se pudo conocer su noción de gestión por competencias, lo que proporcionó una visión de cómo optimizar el DO a través de los resultados a favor de las competencias gerenciales.

Basándose en los datos del compendio estadístico 2016, la población de estudio se segmentó de la administración central. De un total de 1275 colaboradores entre autoridades, directores, jefes y trabajadores, se obtuvo la muestra representativa de 296 administrativos. Se utilizó el método de muestreo probabilístico estratificado y se recurrió a información de fuentes secundarias para la selección de la muestra.

\section{RESULTADOS}

Para el presente trabajo de investigación, se utilizó un cuestionario tipo escala de Likert como técnica de recolección de información, el cual fue aplicado al personal administrativo con el propósito de recopilar sus opiniones respecto a las competencias gerenciales. Asimismo, se incluyeron 5 competencias específicas gerenciales del libro de Alles (2009) en la elaboración del cuestionario:

Conducción de personas: se entiende como la capacidad para valorar y elegir al personal que trabaja en la universidad. El resultado obtenido según la encuesta se muestra en la Tabla 1.

En la Tabla 1, se aprecia que el $56.4 \%$ de los encuestados manifiesta que "casi siempre" se cumplen con las acciones necesarias para una buena conducción del personal.

Empowerment: comprende la capacidad de alcanzar las metas de la UNMSM por medio de la gestión

Tabla 1. Resultados de la encuesta "Conducción de personas".

\begin{tabular}{|l|c|c|c|c|c|}
\hline \multirow{2}{*}{ Conducción de personas } & \multicolumn{4}{|c|}{ Porcentaje válido } \\
\cline { 2 - 5 } & Siempre & Casi siempre & A veces & Casi nunca & Nunca \\
\hline $\begin{array}{l}\text { Establece los contextos para la valoración y/o elección } \\
\text { del personal administrativo }\end{array}$ & $22.0 \%$ & $39.5 \%$ & $29.7 \%$ & $3.7 \%$ & $4.7 \%$ \\
\hline $\begin{array}{l}\text { Guía y motiva a su personal para aumentar la efectividad de } \\
\text { sus funciones }\end{array}$ & $20.3 \%$ & $48.3 \%$ & $28.0 \%$ & $2.7 \%$ & $0.7 \%$ \\
\hline $\begin{array}{l}\text { Identifica las necesidades del personal para su desarrollo } \\
\text { personal }\end{array}$ & $15.2 \%$ & $45.3 \%$ & $37.2 \%$ & $2.0 \%$ & $0.3 \%$ \\
\hline $\begin{array}{l}\text { Instituye y conserva instrucciones de monitoreo y valoración } \\
\text { de desempeño de su personal }\end{array}$ & $29.1 \%$ & $55.4 \%$ & $15.2 \%$ & - & $0.3 \%$ \\
\hline $\begin{array}{l}\text { Ayuda a que su personal reconozca sus problemas y los } \\
\text { motiva a mejorar }\end{array}$ & $19.9 \%$ & $55.1 \%$ & $23.3 \%$ & $1.0 \%$ & $0.7 \%$ \\
\hline Cuenta con personal que se ajusta al perfil del trabajo & $31.4 \%$ & $56.4 \%$ & $11.1 \%$ & $0.3 \%$ & $0.3 \%$ \\
\hline $\begin{array}{l}\text { Mantiene una buena relación laboral con su personal admi- } \\
\text { nistrativo }\end{array}$ & $30.7 \%$ & $48.0 \%$ & $18.6 \%$ & - & $2.7 \%$ \\
\hline $\begin{array}{l}\text { Cuando deja alguna labor al personal, comprueba que ten- } \\
\text { gan los recursos necesarios }\end{array}$ & $21.6 \%$ & $25.7 \%$ & $19.3 \%$ & $5.7 \%$ & $0.7 \%$ \\
\hline $\begin{array}{l}\text { Administra y se asegura de que el trabajo administrativo se } \\
\text { ejecute adecuadamente }\end{array}$ & $34.8 \%$ & $45.9 \%$ & $15.2 \%$ & $3.0 \%$ & $0.7 \%$ \\
\hline $\begin{array}{l}\text { Dirige con compromiso las unidades de producción de su } \\
\text { oficina }\end{array}$ & $30.1 \%$ & $49.0 \%$ & $17.6 \%$ & $2.0 \%$ & - \\
\hline
\end{tabular}

Fuente: Elaboración propia. 
holística del trabajo y el desarrollo de los valores mediante una apropiada comunicación entre los canales de información para obtener mejores decisiones. Los resultados obtenidos según la encuesta se muestran en la Tabla 2.

En la Tabla 2, se aprecia que el $58.2 \%$ de los encuestados manifiesta que "casi siempre" se cumplen con las acciones necesarias para gestionar el trabajo en equipo, a través de una apropiada comunicación entre los canales de información.
Liderazgo ejecutivo: comprende las labores principales del equipo de gestión de la UNMSM para la realización de cambios estratégicos, conducción de equipos y manejo adecuado de los conflictos. Los resultados obtenidos según la encuesta se muestran en la Tabla 3.

En la Tabla 3, se aprecia que el $60 \%$ de los encuestados manifiesta que "casi siempre" se cumple con las acciones necesarias para motivar al equipo de

Tabla 2. Resultados de la encuesta "Empowerment".

\begin{tabular}{|c|c|c|c|c|c|}
\hline \multirow{2}{*}{ Empowerment } & \multicolumn{5}{|c|}{ Porcentaje válido } \\
\hline & Siempre & Casi siempre & A veces & Casi nunca & Nunca \\
\hline $\begin{array}{l}\text { Promueve las virtudes de la universidad y un ambiente } \\
\text { confiable para el cumplimiento de los objetivos en su } \\
\text { dependencia }\end{array}$ & $31.0 \%$ & $46.3 \%$ & $19.4 \%$ & $3.4 \%$ & - \\
\hline $\begin{array}{l}\text { Consulta los medios de información para la mejor toma de } \\
\text { decisiones }\end{array}$ & $14.7 \%$ & $58.2 \%$ & $22.6 \%$ & $4.5 \%$ & - \\
\hline $\begin{array}{l}\text { Comunica de manera objetiva los logros y necesidades a su } \\
\text { personal }\end{array}$ & $20.2 \%$ & $51.7 \%$ & $26.0 \%$ & $2.1 \%$ & - \\
\hline $\begin{array}{l}\text { Mantiene una relación eficiente y cordial con el personal de } \\
\text { toda la universidad }\end{array}$ & $17.6 \%$ & $53.7 \%$ & $25.0 \%$ & $3.4 \%$ & $0.3 \%$ \\
\hline $\begin{array}{l}\text { Proporciona las capacitaciones necesarias para el desarrollo } \\
\text { organizacional en la UNMSM }\end{array}$ & $20.7 \%$ & $42.2 \%$ & $26.5 \%$ & $9.9 \%$ & $0.7 \%$ \\
\hline Mantiene la gestión con modelos de calidad & $20.8 \%$ & $52.6 \%$ & $24.6 \%$ & $1.7 \%$ & $0.3 \%$ \\
\hline Mantiene una evaluación constante de su personal & $29.4 \%$ & $35.1 \%$ & $28.0 \%$ & $6.1 \%$ & $1.4 \%$ \\
\hline $\begin{array}{l}\text { Gestiona de adecuadamente los recursos que la universidad le } \\
\text { brinda para poder lograr los resultados de su dependencia }\end{array}$ & $25.3 \%$ & $47.6 \%$ & $19.6 \%$ & $7.4 \%$ & - \\
\hline $\begin{array}{l}\text { Califica las competencias principales de sus colaboradores } \\
\text { para incrementarlas }\end{array}$ & $33.9 \%$ & $46.4 \%$ & $14.6 \%$ & $4.7 \%$ & $0.3 \%$ \\
\hline
\end{tabular}

Fuente: Elaboración propia.

Tabla 3. Resultados de la encuesta "Liderazgo ejecutivo".

\begin{tabular}{|c|c|c|c|c|c|}
\hline \multirow{2}{*}{ Liderazgo ejecutivo } & \multicolumn{5}{|c|}{ Porcentaje válido } \\
\hline & Siempre & Casi siempre & A veces & Casi nunca & Nunca \\
\hline $\begin{array}{l}\text { Practica el liderazgo y gestiona el cambio, se interrelacio- } \\
\text { na con su personal }\end{array}$ & $27.2 \%$ & $49.1 \%$ & $21.6 \%$ & $1.7 \%$ & $0.3 \%$ \\
\hline $\begin{array}{l}\text { Se expresa adecuadamente y entiende el punto de vista de los } \\
\text { demás }\end{array}$ & $20.8 \%$ & $55.4 \%$ & $19.7 \%$ & $3.8 \%$ & $0.3 \%$ \\
\hline $\begin{array}{l}\text { Utiliza información precisa y consistente para tomar decisiones } \\
\text { pertinentes a fin de lograr los objetivos }\end{array}$ & $21.4 \%$ & $60.0 \%$ & $18.3 \%$ & $0.3 \%$ & - \\
\hline Resuelve conflictos en el momento oportuno & $22.3 \%$ & $50.0 \%$ & $25.0 \%$ & $2.7 \%$ & - \\
\hline $\begin{array}{l}\text { Fomenta la participación de las principales autoridades en la } \\
\text { ejecución de un proyecto educativo institucional }\end{array}$ & $23.8 \%$ & $42.5 \%$ & $26.9 \%$ & $6.8 \%$ & - \\
\hline $\begin{array}{l}\text { Gestiona las habilidades necesarias dentro de su dependencia } \\
\text { para un trabajo más eficiente }\end{array}$ & $18.4 \%$ & $44.9 \%$ & $19.7 \%$ & $15.0 \%$ & $2.0 \%$ \\
\hline $\begin{array}{l}\text { Desarrolla más de una idea de solución cuando se presenta } \\
\text { alguna dificultad típica de su dependencia }\end{array}$ & $24.8 \%$ & $51.4 \%$ & $19.7 \%$ & $4.1 \%$ & - \\
\hline Promueve la gestión por resultados en su dependencia & $19.3 \%$ & $52.2 \%$ & $26.4 \%$ & $1.7 \%$ & $0.3 \%$ \\
\hline $\begin{array}{l}\text { Se establecen objetivos y se delegan disposiciones al comuni- } \\
\text { car los problemas de la dependencia }\end{array}$ & $23.5 \%$ & $50.0 \%$ & $24.1 \%$ & $2.0 \%$ & $0.3 \%$ \\
\hline Considera usted que cuenta con competencias gerenciales & $27.6 \%$ & $44.6 \%$ & $25.2 \%$ & $2.7 \%$ & - \\
\hline
\end{tabular}

Fuente: Elaboración propia. 
trabajo, realizar cambios estratégicos y manejar la gestión de conflictos.

Visión estratégica: se entiende como la habilidad de ajustarse a la vanguardia del ambiente educativo, crear alianzas administrativas entre las distintas oficinas de la UNMSM con el propósito de contribuir al cumplimiento de las metas y objetivos estratégicos determinados. Los resultados obtenidos según la encuesta se muestran en la Tabla 4.

En la Tabla 4, se aprecia que el $61.4 \%$ de los encuestados manifiesta que "casi siempre" se cumplen con las acciones necesarias para mantenerse a la vanguardia de los cambios en el contexto educativo.

Liderar con el ejemplo: control emocional que obedece al directivo y/o jefe en la forma en que se desarrolla para motivar los cambios en su dependencia y ser un ejemplo para el personal. Los resultados obtenidos según la encuesta se muestran en la Tabla 5.

En la Tabla 5, se aprecia que el $52.2 \%$ de los encuestados manifiesta que "casi siempre" se cumplen con las acciones necesarias en la gestión del cargo administrativo en lo que respecta al control de las emociones y a motivar los cambios en la oficina.

Tabla 4. Resultados de la encuesta "Visión estratégica".

\begin{tabular}{|c|c|c|c|c|c|}
\hline \multirow{2}{*}{ Visión estratégica } & \multicolumn{5}{|c|}{ Porcentaje válido } \\
\hline & Siempre & Casi siempre & A veces & Casi nunca & Nunca \\
\hline $\begin{array}{l}\text { Considera usted que tiene las habilidades para enfren- } \\
\text { tar los habituales cambios del entorno en el área donde } \\
\text { labora }\end{array}$ & $25.5 \%$ & $56.8 \%$ & $15.3 \%$ & $2.0 \%$ & $0.3 \%$ \\
\hline $\begin{array}{l}\text { Afronta con flexibilidad y adaptabilidad los cambios del entor- } \\
\text { no, aceptándolos de manera positiva y constructiva }\end{array}$ & $26.7 \%$ & $45.3 \%$ & $23.3 \%$ & $4.4 \%$ & $0.3 \%$ \\
\hline $\begin{array}{l}\text { Desarrolla ideas y procesos novedosos para crear opciones de } \\
\text { mejora continua }\end{array}$ & $15.3 \%$ & $61.4 \%$ & $20.3 \%$ & $3.1 \%$ & - \\
\hline $\begin{array}{l}\text { Se generan ventajas estratégicas que contribuyen con los } \\
\text { procesos de la universidad }\end{array}$ & $18.0 \%$ & $56.5 \%$ & $23.5 \%$ & $2.0 \%$ & - \\
\hline $\begin{array}{l}\text { Crea y ejecuta ideas innovadoras que optimicen el rendimiento } \\
\text { de su personal }\end{array}$ & $20.9 \%$ & $53.1 \%$ & $23.6 \%$ & $2.4 \%$ & - \\
\hline $\begin{array}{l}\text { Formula estrategias como valor agregado a sus funciones y } \\
\text { son compartidas con otras dependencias de la UNMSM }\end{array}$ & $22.5 \%$ & $49.1 \%$ & $25.9 \%$ & $2.4 \%$ & - \\
\hline $\begin{array}{l}\text { Dirige, planea y ejecuta el plan estratégico de su oficina, to- } \\
\text { mando en cuenta la optimización de los recursos brindados }\end{array}$ & $31.1 \%$ & $42.2 \%$ & $18.9 \%$ & $7.1 \%$ & $0.7 \%$ \\
\hline $\begin{array}{l}\text { Impulsa las labores pertinentes de la oficina para mejorar sus } \\
\text { procesos administrativos }\end{array}$ & $20.3 \%$ & $60.3 \%$ & $14.9 \%$ & $4.4 \%$ & - \\
\hline
\end{tabular}

Fuente: Elaboración propia.

Tabla 5. Resultados de la encuesta "Liderar con el ejemplo".

\begin{tabular}{|l|c|c|c|c|c|}
\hline \multirow{2}{*}{ Liderar con el ejemplo } & \multicolumn{4}{|c|}{ Porcentaje válido } \\
\cline { 2 - 5 } & Siempre & Casi siempre & A veces & Casi nunca & Nunca \\
\hline $\begin{array}{l}\text { Ejerce una cualidad emprendedora que propicia cambios } \\
\text { estratégicos en su oficina }\end{array}$ & $19.4 \%$ & $51.7 \%$ & $19.7 \%$ & $7.1 \%$ & $2.0 \%$ \\
\hline $\begin{array}{l}\text { Maneja sus emociones y actúa apropiadamente ante distintas } \\
\text { situaciones y personas }\end{array}$ & $20.1 \%$ & $50.7 \%$ & $21.4 \%$ & $5.4 \%$ & $2.4 \%$ \\
\hline $\begin{array}{l}\text { Considera que la práctica continua es significativa para el } \\
\text { desempeño de las tareas del directivo }\end{array}$ & $27.1 \%$ & $42.0 \%$ & $22.7 \%$ & $8.1 \%$ & - \\
\hline $\begin{array}{l}\text { ¿La universidad le facilita cursos de capacitación para desa- } \\
\text { rrollar adecuadamente su trabajo directivo? }\end{array}$ & $11.2 \%$ & $42.5 \%$ & $35.7 \%$ & $10.5 \%$ & - \\
\hline $\begin{array}{l}\text { Identifica y ejecuta las gestiones necesarias para alcanzar sus } \\
\text { metas de carrera }\end{array}$ & $18.6 \%$ & $52.2 \%$ & $25.4 \%$ & $3.1 \%$ & $0.7 \%$ \\
\hline Prioriza el bien social sobre los beneficios particulares & $25.3 \%$ & $38.9 \%$ & $24.6 \%$ & $9.2 \%$ & $2.0 \%$ \\
\hline $\begin{array}{l}\text { Es profesionalmente responsable y desarrolla sus funciones } \\
\text { de gestión con principios éticos }\end{array}$ & $36.2 \%$ & $47.8 \%$ & $12.6 \%$ & $1.7 \%$ & $1.7 \%$ \\
\hline
\end{tabular}

Fuente: Elaboración propia. 
Adicionalmente, para saber qué competencias consideran en la UNMSM, se formuló la siguiente pregunta: ¿Qué competencias principales corresponderían a un directivo para ejercer un cargo de gerencia universitaria? El resultado obtenido según la encuesta se muestra en la Figura 1.

En la Figura 1, se muestra la lista de las habilidades que, según los encuestados, sus superiores deberían poseer a fin de desarrollar mejor sus funciones; la de liderazgo es la que más sobresalta, con un $11.61 \%$.

Una vez analizada la información, se comprobó la existencia de ciertas deficiencias y problemas que deben abordarse en conjunto con el personal administrativo. Es ventajoso elaborar un modelo de gestión del talento humano por competencias que contribuya con el desempeño de los responsables administrativos, con la finalidad de optimizar la calidad y la eficiencia de los procesos administrativos. De ahí la importancia de establecer competencias específicas por cargo a través de un análisis funcional de cada perfil que permita definir las competencias apropiadas.

\section{Prueba de hipótesis}

Según Hernández et al. (2010), las hipótesis señalan lo que buscamos demostrar, de manera que se definen como posibles aclaraciones del fenómeno de estudio. Su formulación debe ser desarrollada a manera de proposición.

\section{Hipótesis general}

$\mathbf{H}_{1}$ : Existe relación entre la gestión del talento humano por competencias y el desarrollo organizacional en la UNMSM.

$\mathbf{H}_{0}$ : No existe relación entre la gestión del talento humano por competencias y el desarrollo organizacional en la UNMSM.

Los datos presentados en las Tablas 6 y 7 apoyan la sustentación de la hipótesis en mención.

El valor de significancia es $0.000<0.05$ con lo cual se rechaza la $\mathbf{H}_{0}$ y se acepta la $\mathbf{H}_{1}$.

\section{Hipótesis específica 1}

$\mathbf{H}_{1}$ : Existe correlación en el desarrollo de la gestión por competencias y la competitividad en la UNMSM.

$\mathbf{H}_{\mathbf{0}}$ : No existe correlación en el desarrollo de la gestión por competencias y la competitividad en la UNMSM.

Los datos presentados en las Tablas 8 y 9 apoyan la sustentación de la hipótesis en mención. El valor de significancia es $0.000<0.05$ con lo cual se rechaza la $\mathbf{H}_{0}$ y se acepta la $\mathbf{H}_{1}$.

\section{Hipótesis específica 2}

$\mathbf{H}_{1}$ : Existe correlación entre el sistema de gestión por competencias gerenciales y la mejora de perfiles en la UNMSM.

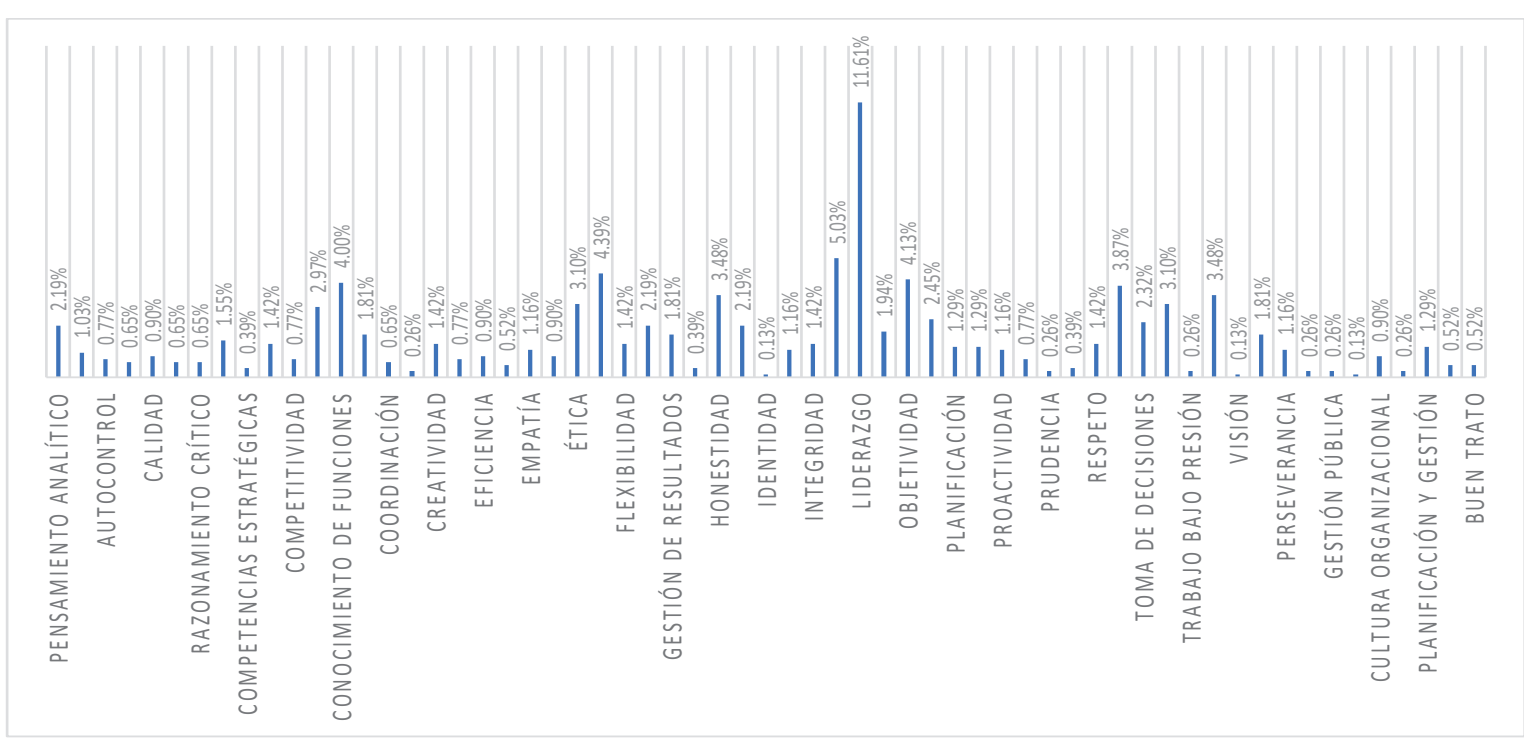

Figura 1. Resultado de encuesta "Competencias clave".

Fuente: Elaboración propia. 
Tabla 6. Cruce de datos - Hipótesis general.

\begin{tabular}{|c|c|c|c|c|c|c|c|}
\hline \multicolumn{8}{|c|}{$\begin{array}{c}\text { Se plantean ventajas estratégicas que ayudan los procesos de la universidad * ¿Considera usted que cuenta con compe- } \\
\text { tencias gerenciales? }\end{array}$} \\
\hline & & & \multicolumn{4}{|c|}{$\begin{array}{l}\text { Planteamiento de ventajas estratégicas para el progre- } \\
\text { so de la universidad }\end{array}$} & \multirow[t]{2}{*}{ Total } \\
\hline & & & Casi nunca & A veces & Casi siempre & Siempre & \\
\hline \multirow{8}{*}{$\begin{array}{l}\text { Competencias } \\
\text { generales }\end{array}$} & \multirow{2}{*}{ Casi nunca } & Recuento & 3 & 0 & 1 & 2 & 6 \\
\hline & & Recuento esperado & 0.2 & 1.5 & 2.7 & 1.7 & 6.0 \\
\hline & \multirow{2}{*}{ A veces } & Recuento & 1 & 28 & 23 & 16 & 68 \\
\hline & & Recuento esperado & 1.9 & 16.8 & 30.5 & 18.9 & 68.0 \\
\hline & \multirow{2}{*}{ Casi siempre } & Recuento & 3 & 37 & 90 & 35 & 165 \\
\hline & & Recuento esperado & 4.5 & 40.7 & 74.0 & 45.8 & 165.0 \\
\hline & \multirow{2}{*}{ Siempre } & Recuento & 1 & 7 & 17 & 28 & 53 \\
\hline & & Recuento esperado & 1.5 & 13.1 & 23.8 & 14.7 & 53.0 \\
\hline \multirow{2}{*}{\multicolumn{2}{|c|}{ Total }} & Recuento & 8 & 72 & 131 & 81 & 292 \\
\hline & & Recuento esperado & 8.0 & 72.0 & 131.0 & 81.0 & 292.0 \\
\hline
\end{tabular}

Fuente: Elaboración propia.

Tabla 7. Prueba de chi-cuadrado - Hipótesis general.

\begin{tabular}{|l|c|c|c|}
\hline \multicolumn{3}{|c|}{ Pruebas de chi-cuadrado } & Significación asintótica (bilateral) \\
\hline Chi-cuadrado de Pearson & Valor & gl & 0.000 \\
\hline Razón de verosimilitud & $85.478^{\text {a }}$ & 9 & 0.000 \\
\hline Asociación lineal por lineal & 47482 & 9 & 0.000 \\
\hline N de casos válidos & 17990 & 1 & \\
\hline
\end{tabular}

Fuente: Elaboración propia.

a. 7 casillas $(43.8 \%)$ han esperado un recuento menor que 5. El recuento mínimo esperado es 0.16 .

Tabla 8. Cruce de datos - Hipótesis específica 1.

Considera usted que tiene las habilidades para enfrentar los habituales cambios del entorno en el área donde labora * Se plantean ventajas estratégicas que ayudan los procesos de la universidad

Planteamiento de ventajas estratégicas para el progreso de la universidad

\begin{tabular}{|l|l|}
\hline \multirow{4}{*}{$\begin{array}{l}\text { Casi } \\
\text { siempre }\end{array}$} \\
\cline { 2 - 3 } & Siempre \\
\cline { 2 - 3 } &
\end{tabular}

Total

Fuente: Elaboración propia.

\begin{tabular}{|l|l|c|c|c|c|c|}
\multicolumn{2}{|c|}{} & \multicolumn{3}{c|}{ Grado de capacitación } & \multirow{2}{*}{ Total } \\
\cline { 3 - 7 } \multicolumn{2}{c|}{} & $\begin{array}{c}\text { Casi } \\
\text { nunca }\end{array}$ & A veces & $\begin{array}{c}\text { Casi } \\
\text { siempre }\end{array}$ & Siempre & \\
\hline \multirow{2}{*}{ Nunca } & Recuento & 0 & 0 & 1 & 0 & 1 \\
\cline { 2 - 7 } & Recuento esperado & 0.0 & 0.2 & 0.6 & 0.2 & 1.0 \\
\hline \multirow{2}{*}{$\begin{array}{l}\text { Casi } \\
\text { nunca }\end{array}$} & Recuento & 0 & 4 & 2 & 0 & 6 \\
\cline { 2 - 7 } & Recuento esperado & 0.1 & 1.4 & 3.4 & 1.1 & 6.0 \\
\hline \multirow{3}{*}{ A veces } & Recuento & 4 & 12 & 27 & 2 & 45 \\
\cline { 2 - 7 } & Recuento esperado & 0.9 & 10.6 & 25.3 & 8.2 & 45.0 \\
\hline \multirow{2}{*}{$\begin{array}{l}\text { Casi } \\
\text { siempre }\end{array}$} & Recuento & 1 & 37 & 102 & 26 & 166 \\
\cline { 2 - 7 } & Recuento esperado & 3.4 & 39.2 & 93.2 & 30.1 & 166.0 \\
\hline \multirow{2}{*}{\begin{tabular}{l} 
Siempre \\
\cline { 2 - 7 }
\end{tabular}} & Recuento & 1 & 16 & 32 & 25 & 74 \\
\cline { 2 - 7 } & Recuento esperado & 1.5 & 17.5 & 41.6 & 13.4 & 74.0 \\
\cline { 2 - 7 } & Recuento & $\mathbf{6}$ & $\mathbf{6 9}$ & $\mathbf{1 6 4}$ & $\mathbf{5 3}$ & $\mathbf{2 9 2}$ \\
\cline { 2 - 7 } & Recuento esperado & $\mathbf{6 . 0}$ & $\mathbf{6 9 . 0}$ & $\mathbf{1 6 4 . 0}$ & $\mathbf{5 3 . 0}$ & $\mathbf{2 9 2 . 0}$ \\
\hline
\end{tabular}


$\mathbf{H}_{0}$ : No existe correlación entre el sistema de gestión por competencias gerenciales y la mejora de perfiles en la UNMSM.

Los datos presentados en las Tablas 10 y 11 apoyan la sustentación de la hipótesis en mención.

El valor de significancia es $0.002<0.05$ con lo cual se rechaza la $\mathbf{H}_{0}$ y se acepta la $\mathbf{H}_{1}$.

\section{DISCUSIÓN}

Después de analizar la información recaudada, se observa que la gestión por competencias y el desarrollo organizacional están relacionadas. Asimismo, se encontraron algunas faltas y problemas que se deben solucionar con los servidores administrativos de la sede central de la universidad; de modo que

Tabla 9. Prueba de Chi-cuadrado - Hipótesis específica 1.

\begin{tabular}{|l|c|c|c|}
\hline \multicolumn{3}{|c|}{ Pruebas de chi-cuadrado } \\
\hline & Valor & gl & Significación asintótica (bilateral) \\
\hline Chi-cuadrado de Pearson & $38.121^{\mathrm{a}}$ & 12 & 0.000 \\
\hline Razón de verosimilitud & 34463.000 & 12 & 0.001 \\
\hline Asociación lineal por lineal & 15825.000 & 1 & 0.000 \\
\hline N de casos válidos & 292 & & \\
\hline
\end{tabular}

Fuente: Elaboración propia.

a. 11 casillas $(55.0 \%)$ han esperado un recuento menor que 5 . El recuento mínimo esperado es 0.02 .

Tabla 10. Cruce de datos - Hipótesis específica 2.

\begin{tabular}{|c|c|c|c|c|c|c|c|}
\hline \multicolumn{8}{|c|}{$\begin{array}{c}\text { Practica el liderazgo y gestiona el cambio interrelaciona con su personal * Se plantea ventajas estratégicas que ayudan los } \\
\text { procesos de la universidad }\end{array}$} \\
\hline & & & \multicolumn{4}{|c|}{ Liderazgo y gestión de cambio } & \multirow{2}{*}{ Total } \\
\hline & & & Casi nunca & A veces & Casi siempre & Siempre & \\
\hline \multirow{10}{*}{$\begin{array}{l}\text { Planteamiento de ventajas } \\
\text { estratégicas para progreso } \\
\text { de la universidad }\end{array}$} & \multirow{2}{*}{ Nunca } & Recuento & 0 & 1 & 4 & 0 & 5 \\
\hline & & Recuento esperado & 0.0 & 0.11 & 2.9 & 0.9 & 5.0 \\
\hline & \multirow{2}{*}{ Casi Nunca } & Recuento & 1 & 2 & 2 & 0 & 5 \\
\hline & & Recuento esperado & 0.1 & 1.2 & 2.8 & 0.9 & 5.0 \\
\hline & \multirow{2}{*}{ A veces } & Recuento & 1 & 23 & 35 & 3 & 62 \\
\hline & & Recuento esperado & 1.3 & 14.4 & 35.0 & 11.4 & 62.0 \\
\hline & \multirow{2}{*}{ Casi siempre } & Recuento & 2 & 30 & 80 & 27 & 139 \\
\hline & & Recuento esperado & 2.9 & 32.2 & 78.4 & 25.5 & 139.0 \\
\hline & \multirow{2}{*}{ Siempre } & Recuento & 2 & 11 & 42 & 23 & 78 \\
\hline & & Recuento esperado & 1.6 & 18.1 & 44.0 & 14.3 & 78.0 \\
\hline \multirow{2}{*}{\multicolumn{2}{|c|}{ Total }} & Recuento & 6 & 67 & 163 & 53 & 289 \\
\hline & & Recuento esperado & 6.0 & 67.0 & 163.0 & 53.0 & 289.0 \\
\hline
\end{tabular}

Fuente: Elaboración propia.

Tabla 11. Prueba de chi-cuadrado - Hipótesis específica 2.

\begin{tabular}{|l|c|c|c|}
\hline \multicolumn{3}{|c|}{ Pruebas de chi-cuadrado } \\
\hline & Valor & gl & Significación asintótica (bilateral) \\
\hline Chi-cuadrado de Pearson & $36.098^{\text {a }}$ & 15 & 0.002 \\
\hline Razón de verosimilitud & 34473.000 & 15 & 0.003 \\
\hline Asociación lineal por lineal & 13877.000 & 1 & 0.000 \\
\hline N de casos válidos & 289 & & \\
\hline
\end{tabular}

Fuente: Elaboración propia.

a. 15 casillas $(62.5 \%$ ) han esperado un recuento menor que 5 . El recuento mínimo esperado es 0.02 . 
es necesario implementar un modelo de gestión por competencias en la administración de recursos humanos que permita que las personas que ocupan los puestos de cargos administrativos logren un mejor desempeño. La selección del talento humano es una acción que tiene como propósito encontrar a la persona que reúna las competencias, conocimientos y otros elementos, como sus grados académicos, necesarios para conseguir un puesto específico de trabajo dentro de una organización.

Acerca del proceso de selección que utiliza la universidad, se puede indicar que se han diseñado instrumentos con los cuales puede abastecerse eficientemente de personal idóneo. Por otra parte, los equipos de gestión de cada dependencia buscan establecer procesos de estimulación y alineación para así instaurar una mejora constante en el desempeño de las funciones. Con la atención de estas necesidades, el personal administrativo busca mantener el desarrollo organizacional dentro de la administración central.

Es fundamental que la oficina general de recursos humanos agilice el proceso de implementación de los lineamientos del régimen SERVIR para la aplicación del manual de perfiles de puesto por competencias gerenciales en los cargos administrativos. Asimismo, considerando cada uno de los indicadores valorados en este estudio, se puede precisar que a pesar de que se realizan las actividades determinadas por cargo, hay situaciones a las que se les debe prestar más atención a fin de levantar las observaciones.

Como resultado del análisis de datos y para contribuir con el objetivo general, se planteó una propuesta de diseño del perfil del cargo basado en competencias (ver Anexo 1). Esta propuesta consigna las ocupaciones y las competencias necesarias para el desarrollo adecuado de funciones. Asimismo, contribuye a la mejora de los formatos de SERVIR en la universidad mediante la introducción de competencias fundamentales, la mejora del nivel de desarrollo por desempeño de funciones, la adecuación del personal al puesto de trabajo, una propuesta de diccionario de competencias gerenciales y la adecuada descripción de perfil de puestos.

En la universidad, la utilización de una guía de gestión por competencias beneficiaría el desarrollo organizacional tal como lo menciona Rodríguez (2015):

Mejora la precisión para determinar el nivel de ajuste y potencial de una persona para diferentes trabajos, de tal forma que los planes de desarrollo puedan relacionarse mejor con las áreas donde realmente se necesita, tal como se puede corroborar en los estudios que sobre la materia ha desarrollado Alles (2009), en los que da cuenta de la importancia de los programas de desarrollo de las personas bajo el modelo de competencias y su relevancia para el crecimiento y continuidad de las organizaciones. (pág. 358)

Asimismo, es necesario plantear los procesos de evaluación de desempeño para los cargos administrativos a fin de monitorear su desarrollo y, con la información obtenida, proponer capacitaciones. Para que las organizaciones se mantengan a la vanguardia deben actualizar sus procesos y adquirir nuevos conceptos que favorezcan el desarrollo de tareas de los trabajadores, para así optimizar los recursos y el adecuado desarrollo organizacional.

\section{CONCLUSIONES}

- Debido a que existe correlación entre la gestión por competencias y el desarrollo organizacional, el rol de la oficina de recursos humanos en la universidad es fundamental para atraer al mejor talento para los cargos administrativos, basándose en una gestión por competencias que influye en el desarrollo organizacional.

- Se observa un bajo nivel de apoyo en lo que respecta a las necesidades del personal. Ello se puede comprobar en el ítem de percepción de apoyo para su desarrollo dentro de la universidad, en donde el 32.6\% manifestó que no sentía el apoyo necesario para realizar sus funciones satisfactoriamente.

- La universidad no ha establecido medidas específicas en sus formatos de selección de personal que acojan el concepto de competencias para cada perfil de puesto de trabajo.

\section{RECOMENDACIONES}

- Es de rigor crear elementos que ayuden a medir las competencias establecidas durante el desarrollo de las tareas y así poder implementar los procesos requeridos para potenciar las habilidades gerenciales. Se debe buscar una mayor competitividad en el cumplimiento de objetivos y la misión institucional a fin de alcanzar el éxito académico. 
- Diseñar un documento donde se describan y especifiquen las tareas y compromisos esenciales del perfil del cargo, que incluya la evaluación de las competencias que se requieren.

- Los presupuestos de capacitación deben ser utilizados para preparar a los miembros del equipo administrativo, de modo que puedan desarrollar con eficiencia y eficacia sus funciones. Asimismo, se debe evaluar su desempeño de manera participativa.

\section{REFERENCIAS BIBLIOGRÁFICAS}

[1] Alles, M. (2008). Dirección Estratégica de Recursos Humanos Gestión por Competencias. Buenos Aires, Argentina: Ediciones Granica S.A.

[2] Alles, M. (2009). Diccionario de competencias: La trilogía. Las 60 competencias más utilizadas. Buenos Aires, Argentina: Ediciones Granica S.A.

[3] Becker, G. (1964). Human Capital. Nueva York, Estados Unidos: Columbia University.

[4] Chiavenato, I. (2002). Gestión del Talento Humano ( $2^{\mathrm{a}}$ ed.). Santafé de Bogotá, Colombia: McGraw Hill.

[5] Chiavenato, I. (2006). Introducción a la Teoría General de la Administración ( $7^{a}$ ed.). México D.F., México: McGraw Hill.

[6] De la Cruz, G. (1999). Problemas y principios para el desarrollo de las organizaciones modernas. Gestión en el Tercer Milenio, 2(3), 45-54.

[7] Delgado, L. (2002). La Gestión Universitaria. En C. Aljovín y C. Germaná(Eds.), La Universidad en el Perú (págs. 81-88). Lima, Perú: Fondo Editorial UNMSM.

[8] Directiva $\mathrm{N}^{\circ}$ 001-2016-SERVIR/GDSRH. Normas para la Gestión del Proceso de Diseño de Puestos y Formulación del Manual de Perfiles de Puesto - MPP. Diario Oficial El Peruano (2016).
[9] Espinoza N. (2000). Gerencia Universitaria: Universidad peruana y tercer milenio. Perú, Lima: Editorial San Marcos.

[10] Fernández L. (2008). Análisis de la gestión universitaria en la universidad pública peruana [artículo]. VIII Coloquio Internacional sobre Gestión Universitaria en América del Sur, Asunción, Paraguay.

[11] Fernández J. (2006). Gestión porcompetencias. Madrid, España: Pearson Educación S.A.

[12] Hernández R., Fernández C., y Baptista P. (2010). Metodología de la Investigación. Bogotá, Colombia: McGraw Hill.

[13] McClelland, D. (1973). Testing for Competence Rather Than for "Intelligence". American Psychologist, 28(1), 1-14.

[14] Resolución Rectoral $\quad N^{\circ}$ 01206-R-11. Reglamento de Organizaciones y Funciones de la Universidad Nacional Mayor de San Marcos (2011).

[15] Rodríguez, J. (2015). Implementación del modelo de gestión de talento humano por competencias en una universidad de Lima Metropolitana. Propósitos y Representaciones, 3(2), 319-401

[16] Sánchez, G. (2009). El Desarrollo Organizacional: Una Estrategia de Cambio para las Instituciones Documentales. Anales de Documentación, (12),235-254. ISSN: 15752437.

[17] Spencer L., y Spencer S. (1993). Competence at work: Models for Superior Performance. Nueva York, Estados Unidos: Wiley \& Sons. 


\section{ANEXO}

Anexo 1. Perfil de competencias propuesto.

\begin{tabular}{|l|l|}
\hline COMPETENCIAS ESPECÍFICAS GERENCIALES & $\begin{array}{l}\text { Capacidad para dirigir al personal administrativo. Capacidad para generar } \\
\text { y desarrollar el talento de su personal y brindar retroalimentación. }\end{array}$ \\
\hline Conducción de personas & $\begin{array}{l}\text { Capacidad para trabajar con su personal administrativo en equipo. } \\
\text { Capacidad para desarrollar y ejercer objetivos de desempeño orientados } \\
\text { a mejorar y afianzar el talento de su personal. Capacidad para obtener } \\
\text { buenos resultados a través de la integración de su personal administra- } \\
\text { tivo aprovechando sus diversidades y dándole un valor a los procesos } \\
\text { administrativos. }\end{array}$ \\
\hline Liderazgo ejecutivo & $\begin{array}{l}\text { Capacidad para desarrollarse y trabajar con los diferentes jefes de otras } \\
\text { dependencias a fin de crear un mejor clima organizacional y compromiso } \\
\text { para su gestión. }\end{array}$ \\
\hline Visión estratégica & $\begin{array}{l}\text { Habilidad para anticipar los cambios y saber comprenderlos. Capacidad } \\
\text { para proponer mejoras sobre aspectos del entorno administrativo. }\end{array}$ \\
\hline Liderazgo con el ejemplo & $\begin{array}{l}\text { Habilidad de difundir estrategias y valores mediante un prototipo de } \\
\text { conducción de trabajadores administrativos. Capacidad para promover la } \\
\text { generación de ideas en un entorno agradable. }\end{array}$ \\
\hline
\end{tabular}

\section{COMPETENCIAS ESPECÍFICAS POR DEPENDENCIA}

\section{FUNCIONES}

1. Planear, administrar, regularizar, inspeccionar y examinar los sistemas y métodos administrativos en el entorno universitario.

2. Dirigir procesos administrativos financieros, los procedimientos de suministro y otros servicios en el ambiente de su competencia.

3. Dirigir y fiscalizar los PIP de la universidad y en el abastecimiento de bienes.

4. Elegir las comisiones especiales según los temas de interés a través de Resoluciones Directorales (RD).

5. Aprobar las bases administrativas a través de RD vigentes.

6. Formular opinión técnica relativa en términos administrativos y financieros en administración universitaria.

7. Proponer políticas para el perfeccionamiento de los sistemas administrativos institucionales.

8. Orientar a la Alta Autoridad en cuestiones relativas a los sistemas administrativos y financieros que administra.

9. Coordinar con los entes reguladores de los regímenes administrativos-financieros de la gestión pública.

10. Planificar y ejecutar capacitaciones en la aplicación de sistemas de gestión, finanzas, entre otros, que correspondan a la administración universitaria.

11. Demás tareas que establezca el Órgano Superior.

Tra

Capacidad para dirigir al personal administrativo. Capacidad para generar y desarrollar el talento de su personal y brindar retroalimentación.

Capacidad para desarrollar y ejercer objetivos de desempeño orientados tivo aprovechando sus diversidades y dándole un valor a los procesos administrativos. para su gestión.

Habilidad para anticipar los cambios y saber comprenderlos. Capacidad

Habilidad de difundir estrategias y valores mediante un prototipo de generación de ideas en un entorno agradable.

Nota: Se tomaron como base las ocupaciones de la Dirección General de Administración expuestas en el ROF UNMSM.

Fuente: Elaboración propia. 


\section{Competency-Based Management for Organizational Development at Universidad Nacional Mayor de San Marcos}

Lucía Fernanda Palmira Castillo Arias ${ }^{1}$

Received: 17/06/2019 Accepted: 25/02/2021 Published: 26/07/2021

\begin{abstract}
This study aims to contribute to the improvement of human resources management through a competencybased management model for administrative positions in the central administration that will lead to organizational development at Universidad Nacional Mayor de San Marcos. This is because, on some occasions, people who hold administrative positions lack the training required to manage, and only have technical knowledge, which is not enough to achieve the objectives determined in university's strategic plan. Different bibliographic sources, studies related to the topic, as well as the methodology established by SERVIR were reviewed. The study revealed that no specific measures have been established for the profiles of management positions at the university, so that it is therefore necessary for the human resources office to implement a competencybased management model and enforce the established guidelines. The introduction of a new proposal based on the competencies to the university invites us to redesign the work profiles in terms of performance functions.
\end{abstract}

Keywords: human talent; competencies; management; university.

\section{INTRODUCTION}

At present, public and private universities are using new competency-based management models for human resources to introduce competitive tactics to address global changes and meet the needs of the human intellect. Accordingly, the general objective of this research is to improve human resources management based on competencies to enable organizational development within the university. For this purpose, it is essential to review various bibliographical sources, studies on the subject and the guidelines of the Autoridad Nacional del Servicio Civil (SERVIR) as the governing body of public servants.

This research proposes a study on human resources management in public and private universities based on competencies, using a model that details the competency-based performance profiles for administrative positions that may be used in future research. It further proposes to establish a competency-based management system for the institutional environment of the university that contributes to the elaboration of the position profiles in order to increase administrative productivity.

Peruvian universities have gone through various organizational changes over the years, and it has been observed that decision-makers react to different situations when they occur, rather than anticipating them, triggering political disruptions and/or interventions that result in the instability of organizational climate. Most often, people in management positions lack the necessary managerial skills, but are hired for their academic and/or technical knowledge, which are insufficient to lead teams and make decisions. The way in which university managers perform their functions is directly related to organizational development (OD), hence the importance of ensuring that the members are satisfied with the performance of their functions so that, as a team, they achieve success and institutional objectives. The main function of administrative positions within the public university is the efficient management of its own or ordinary resources.

Degree in Business Management from Universidad Inca Garcilaso de la Vega. Currently working as head of Marketing at the Centro de Informática of the Universidad Nacional Mayor de San Marcos. (Lima, Peru)

ORCID: https://orcid.org/0000-0001-8632-6906

Corresponding author: 1castilloa1@unmsm.edu.pe 


\section{BACKGROUND}

According to Delgado (2002), proper administration consists of effectively and efficiently using the university's resources, namely, all the members of a university, in order to achieve the institution's objectives. Those who manage the university should have an idea about the members of this community. A new form of managing a university should be based on a management team that works with responsibility, diligence and efficiency.

Thus, for proper management, human resources departments should consider using competency standards, as having the most suitable personnel will ensure compliance with institutional objectives as part of organizational development. In addition, an individual's skills to perform the job functions are identified through the achievement of objectives.

From the various bibliographic sources reviewed, it was found that competency-based management is a management prototype that aligns human capital with the essence of the organization, so that workers are professionally oriented, which contributes to the fulfillment/ of the strategic plan.

In this regard, several studies have addressed human resources management in universities. According to one, a competency-based management model should be implemented for the administrative profiles of a university, because a) a competency-based approach supports continuous learning, helps to incorporate training programs and to adapt to the technological advances; b) it specifies the profile of suitable personnel and thus provides an understanding of what is expected of the administrative-teaching staff and how competencies efficiently help in achieving the objective; c) personnel selection should no longer be based solely on academic degrees, but also on the competencies that the personnel suitable for the job profile should have; and d) it allows updating administrative profiles in order to align development plans to the needs of each area (Rodríguez, 2015).

In organizations, human talent management consists of recognizing the value of employees, improving their productivity and retaining them, because they are essential elements for the efficient management of the organization; human capital management is a perceptible factor in organizational culture, especially considering that individuals spend most of their lives working, and organizations depend on them to operate and achieve their objectives (Chiavenato, 2002).
In Human Capital, Becker (1964) focused on the study of information societies, and determined human beings to be the most valuable capital for organizations, on account of their knowledge and skills manifested in their work habits. Thus, Becker considers human talent as the main factor for the performance of today's economies, since human productivity is based on knowledge.

Alles (2008) explains that current business management models promote the development of employees depending on the organization's strategic plans, therefore, all members of the organization can be involved in the organizational strategy and participate in its execution, which leads to the fulfillment of the objectives set.

In Testing for Competence Rather Than for "Intelligence", McClelland (1973) defines the term "competence" as the main trait of human beings, as it is the source of their productivity for the performance of their functions at work.

On the other hand, Spencer and Spencer (1993) define competencies as the main attributes of human beings, which are manifested in their behavior or mindset.

A comprehensive concept of competency-based management suggests that it is a perennial means of integration where the organization incorporates the needs and desires of workers in order to help and support them so that they continue to contribute to the institutional objectives. According to Fernández (2006), human talent management involves the development of new proposals to simplify management of workers and their adaptation to the needs of the company.

Competency-based management represents not only the future, but also the current need for a new workforce generation in organizations. It will identify the main competencies that a person requires to meet the profile of a position, which will allow to maintain high or superior performance levels and incorporate the ideal individual into the organization, which in turn will create competitive advantages for the organization's development.

Several authors have made contributions about OD, which is understood as an administrative tactic that enables the fulfillment of the strategic plan through the proper use of management and human resources tools. De la Cruz (1999) describes organizational development as a procedure that takes into account all the elements related to change in order to devel- 
op a new organizational model, focusing exclusively on the human factor.

Organizational development should focus on establishing the mission, vision, institutional values, tactics and their implementation, to ensure that all employees are able to adapt to continuous change.

Sánchez (2009) states that a proper administrative process, together with the evaluation of personnel performance, achieves the success of the competitive strategy, adding to the premise: human capital and its aspiration to achieve objectives pushes finances and technology into the background.

The classic author on organizational development, Bennis (1997, as cited in Sánchez, 2009), explains that it is a response to change, a complex educational strategy that aims to change management attitudes in the organization and restructure it so that it can adapt to global changes in organizational trends, such as new technologies, markets and business challenges.

Chiavenato (2006) states that OD is a long-term procedure, led by the general management of the organization, that allows the organization's processes to be more effective and contributes to personnel development.

Based on the definitions of OD, the nexus between organizational development and university management can be reshaped into organizational processes, including their theoretical, methodological and operational characteristics, which allow the achievement of institutional objectives.

According to Espinoza (2000):

Hoy en día, la premisa "capacidad gerencial demostrada" en la gerencia universitaria no es un elemento valorativo para componer la estructura gerencial de la universidad, queriendo significar que el personal que ocupa cargos de gestión muchas veces están ahí por cuestiones políticas y/o de poder políti$c o$. [At present, the premise "demonstrated managerial competence" in university management is not considered an important element in the constitution of the university's management structure, thus implying that the personnel in management positions are often there on account of political interests and/or political power.] (p. 87)

In the opinion of Fernández (2008):
La Universidad es un centro generador de conocimiento, y por ello su rol y efecto en el desarrollo de las sociedades y de los países es fundamental. Sin embargo, principalmente en el caso de la universidad pública, existen varios factores que impiden su mayor competitividad y liderazgo; y por lo tanto su aporte en el desarrollo del país. Un factor podría ser la forma de gobierno o gestión universitaria; otra sería su cultura y/o medioambiente organizacional. La Universidad pública peruana presenta diversos problemas que suceden en un entorno interno $y$ externo adversos. Internamente, puede estar la falta de una gestión universitaria que defina e implemente estrategias para la competitividad y calidad; y externamente, el rol del Estado, por su falta de intervención y definición de una política educativa como estrategia de desarrollo y bienestar. [Universities are sources of knowledge, and therefore their role and impact on the development of societies and countries is paramount. Nevertheless, mostly in public universities, several factors interfere with their competitiveness and leadership, and consequently hinder their contribution to the country's development. These factors could be the university governance or management and its organizational culture and/ or environment. Public universities in Peru face a number of problems in an adverse internal and external environment. Internally, there is the lack of a university management that defines and implements strategies for competitiveness and quality; and externally, the role of the State, for its lack of intervention and definition of an educational policy as a strategy for development and welfare.] (p. 3)

The Universidad Nacional Mayor de San Marcos (UNMSM) is an educational entity aimed at perfection and leadership. As such, people involved in its management must comply with the corporate objectives and be resourceful when faced with the constant changes that university management demands. Committed and identified personnel with high levels of competence are required to achieve a stable and continuous organizational development; likewise, it is necessary to provide service quality and promote the achievement of strategic objectives. Therefore, training for administrative positions must be arranged for the improvement of knowledge. 
The Reglamento de Organización y Funciones (ROF) approved with R.R. No. 01206-R-11 (2011) is the official document that recognizes the UNMSM as an educational institution, details its nature and authority, and the general and specific functions of each of its bodies and its administrative units.

In Peru, the management and administrative staff of public educational institutions work based on SERVIR's guidelines, complying with training and continuous development regimes for public servants. This research used the guidelines of the Manual de Perfiles de Puestos [Position Profile Manual] (MPP), approved with Directive No. 001-2016-SERVIR/GDSRH (2016).

\section{METHODOLOGY}

This is a mixed study, qualitative because the information collected expresses quality, and quantitative because information was collected using indicators; data analysis was performed using statistical programs. Its nature is descriptive-correlational, as it aims to measure the relationship between competency-based management and organizational development at the university; and it follows a non-experimental design. Respondents' opinions were also examined based on their answers and observations and thus it was possible to know their concept of competency-based management, which provided an idea of how to optimize OD using the results in favor of managerial competencies.

Based on data from the 2016 statistical compendium, the study population was selected from central management. From a total of 1275 administrative staff, including authorities, directors, heads and workers, a representative sample of 296 was selected. The stratified probability sampling method and information from secondary sources were used to select the sample.

\section{RESULTS}

A Likert scale questionnaire was used as data collection technique, and it was applied to administrative personnel in order to gather their opinions on management competencies. Five specific management competencies from the book by Alles (2009) were included in the questionnaire:

People Management. This is the ability to assess and select the personnel working at the university. The results obtained from the questionnaire are shown in Table 1.

As shown in Table 1,56.4\% of the respondents state that the necessary actions for good personnel management are "almost always" undertaken.

Table 1. Results of Questionnaire "People Management".

\begin{tabular}{|l|c|c|c|c|c|}
\hline \multicolumn{1}{|c|}{ People Management } & \multicolumn{4}{c|}{ Valid Percentage } \\
\cline { 2 - 5 } & Always & Almost Always & Sometimes & Almost Never & Never \\
\hline $\begin{array}{l}\text { Establishes contexts for the evaluation and/or selection of } \\
\text { administrative staff }\end{array}$ & $22.0 \%$ & $39.5 \%$ & $29.7 \%$ & $3.7 \%$ & $4.7 \%$ \\
\hline $\begin{array}{l}\text { Guides and motivates staff members to improve their perfor- } \\
\text { mance effectiveness }\end{array}$ & $20.3 \%$ & $48.3 \%$ & $28.0 \%$ & $2.7 \%$ & $0.7 \%$ \\
\hline Identifies staff needs for personal development & $15.2 \%$ & $45.3 \%$ & $37.2 \%$ & $2.0 \%$ & $0.3 \%$ \\
\hline $\begin{array}{l}\text { Sets and maintains performance monitoring and evaluation } \\
\text { instructions for staff }\end{array}$ & $29.1 \%$ & $55.4 \%$ & $15.2 \%$ & - & $0.3 \%$ \\
\hline $\begin{array}{l}\text { Encourages staff to acknowledge their deficiencies and } \\
\text { motivates them to improve }\end{array}$ & $19.9 \%$ & $55.1 \%$ & $23.3 \%$ & $1.0 \%$ & $0.7 \%$ \\
\hline Staff members fit the job profile & $31.4 \%$ & $56.4 \%$ & $11.1 \%$ & $0.3 \%$ & $0.3 \%$ \\
\hline $\begin{array}{l}\text { Maintains a good working relationship with administrative } \\
\text { staff }\end{array}$ & $30.7 \%$ & $48.0 \%$ & $18.6 \%$ & - & $2.7 \%$ \\
\hline $\begin{array}{l}\text { Verifies that staff members have the necessary resources to } \\
\text { perform tasks assigned }\end{array}$ & $21.6 \%$ & $25.7 \%$ & $19.3 \%$ & $5.7 \%$ & $0.7 \%$ \\
\hline $\begin{array}{l}\text { Manages and ensures that the administrative work is execu- } \\
\text { ted properly }\end{array}$ & $34.8 \%$ & $45.9 \%$ & $15.2 \%$ & $3.0 \%$ & $0.7 \%$ \\
\hline Leads the production units in the office with dedication & $30.1 \%$ & $49.0 \%$ & $17.6 \%$ & $2.0 \%$ & - \\
\hline
\end{tabular}

Source: Prepared by the author. 
Empowerment. It is the ability to achieve UNMSM's goals through holistic work management and development of values based on appropriate communication between information channels to make better decisions. The results obtained from the questionnaire are shown in Table 2.

As shown in Table 2, 58.2\% of the respondents state that the necessary actions for managing teamwork, through appropriate communication between information channels, are "almost always" undertaken.
Executive Leadership. It comprises the main functions of UNMSM's management team for the implementation of strategic changes, team leadership and adequate conflict management. The results obtained from the questionnaire are shown in Table 3.

As shown in Table 3, $60 \%$ of the respondents state that the necessary actions to motivate work teams, carry out strategic changes and manage conflict management are "almost always" undertaken.

Table 2. Results of Questionnaire "Empowerment".

\begin{tabular}{|l|c|c|c|c|c|}
\hline \multicolumn{1}{|c|}{ Empowerment } & \multicolumn{4}{c|}{ Valid Percentage } \\
\cline { 2 - 5 } & Always & Almost Always & Sometimes & Almost Never & Never \\
\hline $\begin{array}{l}\text { Promotes the university's virtues and a secure environment for } \\
\text { the fulfillment of the objectives of the department }\end{array}$ & $31.0 \%$ & $46.3 \%$ & $19.4 \%$ & $3.4 \%$ & - \\
\hline Checks media information for better decision-making & $14.7 \%$ & $58.2 \%$ & $22.6 \%$ & $4.5 \%$ & - \\
\hline Effectively communicates achievements and needs to the staff & $20.2 \%$ & $51.7 \%$ & $26.0 \%$ & $2.1 \%$ & - \\
\hline $\begin{array}{l}\text { Maintains an efficient and cordial relationship with the personnel } \\
\text { of the university }\end{array}$ & $17.6 \%$ & $53.7 \%$ & $25.0 \%$ & $3.4 \%$ & $0.3 \%$ \\
\hline $\begin{array}{l}\text { Provides the necessary training for organizational development } \\
\text { at UNMSM }\end{array}$ & $20.7 \%$ & $42.2 \%$ & $26.5 \%$ & $9.9 \%$ & $0.7 \%$ \\
\hline Uses high quality management models & $20.8 \%$ & $52.6 \%$ & $24.6 \%$ & $1.7 \%$ & $0.3 \%$ \\
\hline Constantly evaluates staff members & $29.4 \%$ & $35.1 \%$ & $28.0 \%$ & $6.1 \%$ & $1.4 \%$ \\
\hline $\begin{array}{l}\text { Appropriately manages the resources provided by the university } \\
\text { in order to meet department objectives }\end{array}$ & $25.3 \%$ & $47.6 \%$ & $19.6 \%$ & $7.4 \%$ & - \\
\hline $\begin{array}{l}\text { Rates the main competencies of employees in order to enhance } \\
\text { them }\end{array}$ & $33.9 \%$ & $46.4 \%$ & $14.6 \%$ & $4.7 \%$ & $0.3 \%$ \\
\hline
\end{tabular}

Source: Prepared by the author.

Table 3. Results of Questionnaire "Executive Leadership".

\begin{tabular}{|c|c|c|c|c|c|}
\hline \multirow{2}{*}{ Executive Leadership } & \multicolumn{5}{|c|}{ Valid Percentage } \\
\hline & Always & Almost Always & Sometimes & Almost Never & Never \\
\hline $\begin{array}{l}\text { Practices leadership, manages change, and interacts with the } \\
\text { staff }\end{array}$ & $27.2 \%$ & $49.1 \%$ & $21.6 \%$ & $1.7 \%$ & $0.3 \%$ \\
\hline $\begin{array}{l}\text { Expresses appropriately and understand the point of view of } \\
\text { others }\end{array}$ & $20.8 \%$ & $55.4 \%$ & $19.7 \%$ & $3.8 \%$ & $0.3 \%$ \\
\hline $\begin{array}{l}\text { Uses accurate and consistent information to make pertinent } \\
\text { decisions to achieve objectives }\end{array}$ & $21.4 \%$ & $60.0 \%$ & $18.3 \%$ & $0.3 \%$ & - \\
\hline Resolves conflicts in a timely manner & $22.3 \%$ & $50.0 \%$ & $25.0 \%$ & $2.7 \%$ & - \\
\hline $\begin{array}{l}\text { Encourages the participation of key authorities in the implemen- } \\
\text { tation of an institutional educational project }\end{array}$ & $23.8 \%$ & $42.5 \%$ & $26.9 \%$ & $6.8 \%$ & - \\
\hline $\begin{array}{l}\text { Manages the necessary competencies within the department for } \\
\text { a more efficient work }\end{array}$ & $18.4 \%$ & $44.9 \%$ & $19.7 \%$ & $15.0 \%$ & $2.0 \%$ \\
\hline $\begin{array}{l}\text { Develops more than one solution idea when faced with a diffi- } \\
\text { culty characteristic of the department }\end{array}$ & $24.8 \%$ & $51.4 \%$ & $19.7 \%$ & $4.1 \%$ & - \\
\hline Promotes results-based management in the department & $19.3 \%$ & $52.2 \%$ & $26.4 \%$ & $1.7 \%$ & $0.3 \%$ \\
\hline $\begin{array}{l}\text { Objectives are set and provisions are delegated when commu- } \\
\text { nicating the department's problems }\end{array}$ & $23.5 \%$ & $50.0 \%$ & $24.1 \%$ & $2.0 \%$ & $0.3 \%$ \\
\hline Do you consider that you possess management competencies? & $27.6 \%$ & $44.6 \%$ & $25.2 \%$ & $2.7 \%$ & - \\
\hline
\end{tabular}

Source: Prepared by the author. 
Strategic Vision. It is the ability to adjust to the changing educational environment, create administrative alliances between the different departments at UNMSM in order to contribute to the fulfillment of the determined strategic objectives. The results obtained according to the questionnaire are shown in Table 4.

As shown in Table 4, 61.4\% of the respondents state that the necessary actions to keep abreast of changes in the educational context are "almost always" undertaken.

Leading by example. It is the emotional control of the manager and/or boss to motivate changes in his or her department and to be an example for the personnel. The results obtained according to the questionnaire is shown in Table 5.

As shown in Table 5, 52.2\% of the respondents state that the necessary actions to manage the administrative position in terms of emotional control and encouraging changes in the department are "almost always" undertaken.

To learn about the competencies considered at UNMSM, the following question was asked: What main competencies should a manager have in order to hold a university management position? The

Table 4. Results of Questionnaire "Strategic Vision".

\begin{tabular}{|c|c|c|c|c|c|}
\hline \multirow{2}{*}{ Strategic Vision } & \multicolumn{5}{|c|}{ Valid Percentage } \\
\hline & Always & Almost Always & Sometimes & Almost Never & Never \\
\hline $\begin{array}{l}\text { Do you consider that you possess the skills to face the frequent } \\
\text { changes in your work environment? }\end{array}$ & $25.5 \%$ & $56.8 \%$ & $15.3 \%$ & $2.0 \%$ & $0.3 \%$ \\
\hline $\begin{array}{l}\text { Faces changes with flexibility and adaptability, accepting them in } \\
\text { a positive and constructive way }\end{array}$ & $26.7 \%$ & $45.3 \%$ & $23.3 \%$ & $4.4 \%$ & $0.3 \%$ \\
\hline $\begin{array}{l}\text { Develops new ideas and processes to create options for conti- } \\
\text { nuous improvement }\end{array}$ & $15.3 \%$ & $61.4 \%$ & $20.3 \%$ & $3.1 \%$ & - \\
\hline $\begin{array}{l}\text { Strategic advantages that contribute to the university's proces- } \\
\text { ses are generated }\end{array}$ & $18.0 \%$ & $56.5 \%$ & $23.5 \%$ & $2.0 \%$ & - \\
\hline $\begin{array}{l}\text { Creates and executes innovative ideas that optimize staff per- } \\
\text { formance }\end{array}$ & $20.9 \%$ & $53.1 \%$ & $23.6 \%$ & $2.4 \%$ & - \\
\hline $\begin{array}{l}\text { Formulates strategies as an added value to their functions and } \\
\text { are shared with other UNMSM departments }\end{array}$ & $22.5 \%$ & $49.1 \%$ & $25.9 \%$ & $2.4 \%$ & - \\
\hline $\begin{array}{l}\text { Leads, plans and executes the strategic plan of the office, taking } \\
\text { into account the optimization of the resources provided }\end{array}$ & $31.1 \%$ & $42.2 \%$ & $18.9 \%$ & $7.1 \%$ & $0.7 \%$ \\
\hline $\begin{array}{l}\text { Drives the relevant work of the office to improve its administrati- } \\
\text { ve processes }\end{array}$ & $20.3 \%$ & $60.3 \%$ & $14.9 \%$ & $4.4 \%$ & - \\
\hline
\end{tabular}

Source: Prepared by the author.

Table 5. Results of Questionnaire "Leading by Example".

\begin{tabular}{|c|c|c|c|c|c|}
\hline \multirow{2}{*}{ Leading by Example } & \multicolumn{5}{|c|}{ Valid Percentage } \\
\hline & Always & Almost Always & Sometimes & Almost Never & Never \\
\hline $\begin{array}{l}\text { Exercises an entrepreneurial mindset that leads to strategic } \\
\text { changes in the department }\end{array}$ & $19.4 \%$ & $51.7 \%$ & $19.7 \%$ & $7.1 \%$ & $2.0 \%$ \\
\hline $\begin{array}{l}\text { Manages emotions and acts appropriately in different situations } \\
\text { and with different people }\end{array}$ & $20.1 \%$ & $50.7 \%$ & $21.4 \%$ & $5.4 \%$ & $2.4 \%$ \\
\hline $\begin{array}{l}\text { Do you consider that continuous practice is relevant for the } \\
\text { performance of the manager's responsibilities? }\end{array}$ & $27.1 \%$ & $42.0 \%$ & $22.7 \%$ & $8.1 \%$ & - \\
\hline $\begin{array}{l}\text { Does the university provide you with training courses to adequa- } \\
\text { tely develop your administrative work? }\end{array}$ & $11.2 \%$ & $42.5 \%$ & $35.7 \%$ & $10.5 \%$ & - \\
\hline $\begin{array}{l}\text { Identifies and executes the necessary steps to achieve career } \\
\text { goals. }\end{array}$ & $18.6 \%$ & $52.2 \%$ & $25.4 \%$ & $3.1 \%$ & $0.7 \%$ \\
\hline Prioritizes the community well-being over individual benefits & $25.3 \%$ & $38.9 \%$ & $24.6 \%$ & $9.2 \%$ & $2.0 \%$ \\
\hline $\begin{array}{l}\text { Management staff members are professionally responsible and } \\
\text { perform their managerial functions with ethical principles }\end{array}$ & $36.2 \%$ & $47.8 \%$ & $12.6 \%$ & $1.7 \%$ & $1.7 \%$ \\
\hline
\end{tabular}

Source: Prepared by the author. 
results obtained from the questionnaire are shown in Figure 1.

A list of the competencies that, according to the respondents, their superiors should possess to better perform their functions is shown in Figure 1. Leadership is the competency that stands out the most with $11.61 \%$.

Upon analyzing the information, certain deficiencies and problems were identified that need to be addressed in collaboration with the administrative staff. It is advisable to develop a competency-based human talent management model that contributes to the performance of administrative leaders in order to optimize the quality and efficiency of administrative processes. Hence the importance of setting specific competencies per position through a functional analysis of each profile that allows determining the proper competencies.

\section{Hypothesis Testing}

According to Hernández et al. (2010), hypotheses state what we intend to demonstrate, so that they are defined as possible explanations of the phenomenon under study. They should be formulated in the form of a proposition.

\section{General Hypothesis}

$H_{1}$ : There is a relationship between competency-based human talent management and organizational development at UNMSM.
$\mathbf{H}_{\mathbf{0}}$ : There is no relationship between competency-based human talent management and organizational development at UNMSM.

Data presented in Tables 6 and 7 support the hypothesis.

The significance level is $0.000<0.05$, therefore the null hypothesis $\mathbf{H}_{0}$ is rejected and $\mathbf{H}_{1}$ is accepted.

\section{Specific Hypothesis 1}

$\mathbf{H}_{1}$ : There is a correlation between the development of competency-based management and competitiveness at UNMSM.

$\mathbf{H}_{0}$ : There is no correlation between the development of competency-based management and competitiveness at UNMSM.

Data presented in Tables 8 and 9 support the hypothesis.

The significance level is $0.000<0.05$, therefore the null hypothesis $\mathbf{H}_{0}$ is rejected and $\mathbf{H}_{1}$ is accepted.

\section{Specific Hypothesis 2}

$\mathbf{H}_{1}$ : There is a correlation between the competency-based management system and the improvement of position profiles at UNMSM.

$\mathbf{H}_{0}$ : There is no correlation between the competency-based management system and the improvement of position profiles at UNMSM.

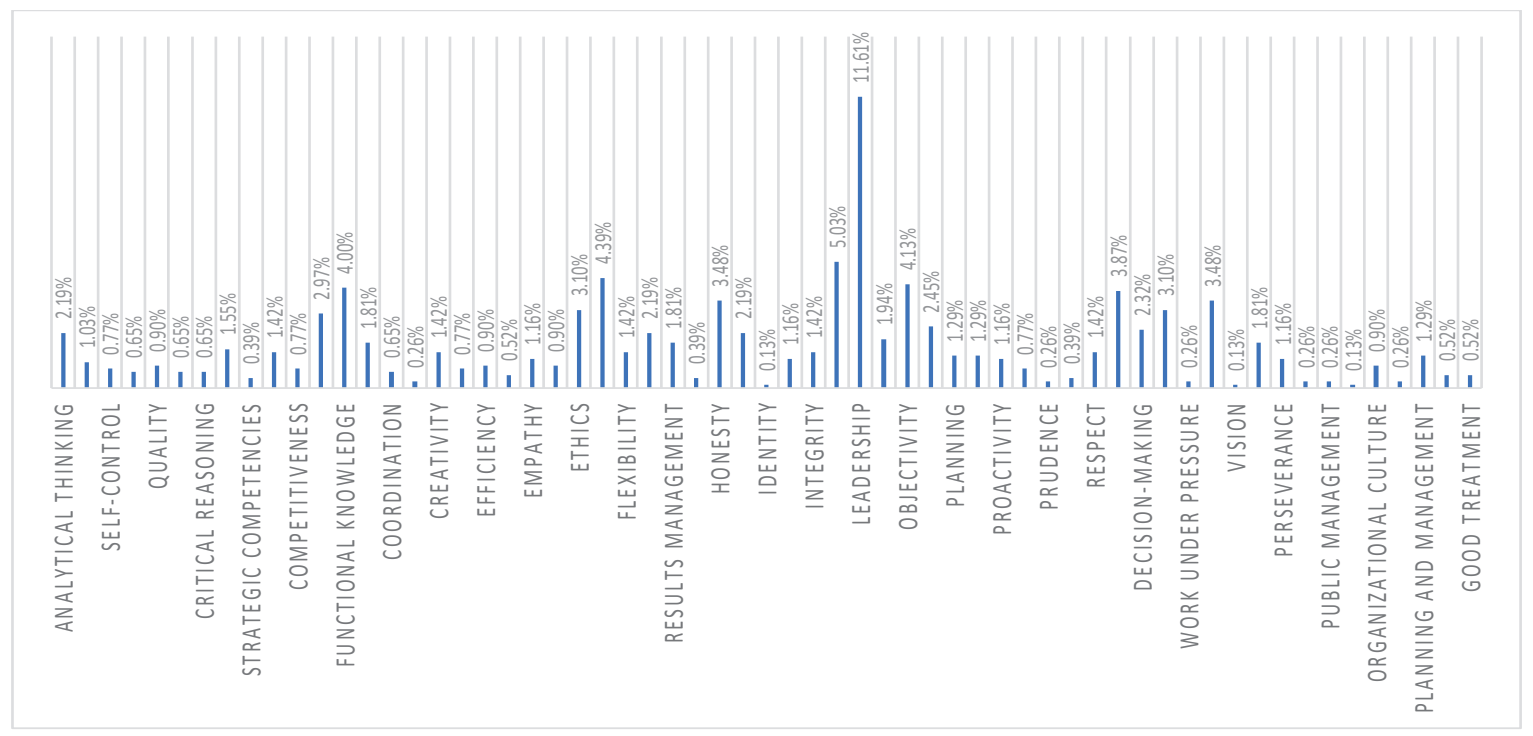

Figure 1. Results of questionnaire "Key Competencies".

Source: Prepared by the author. 
Table 6. Data Cross Checking - General Hypothesis.

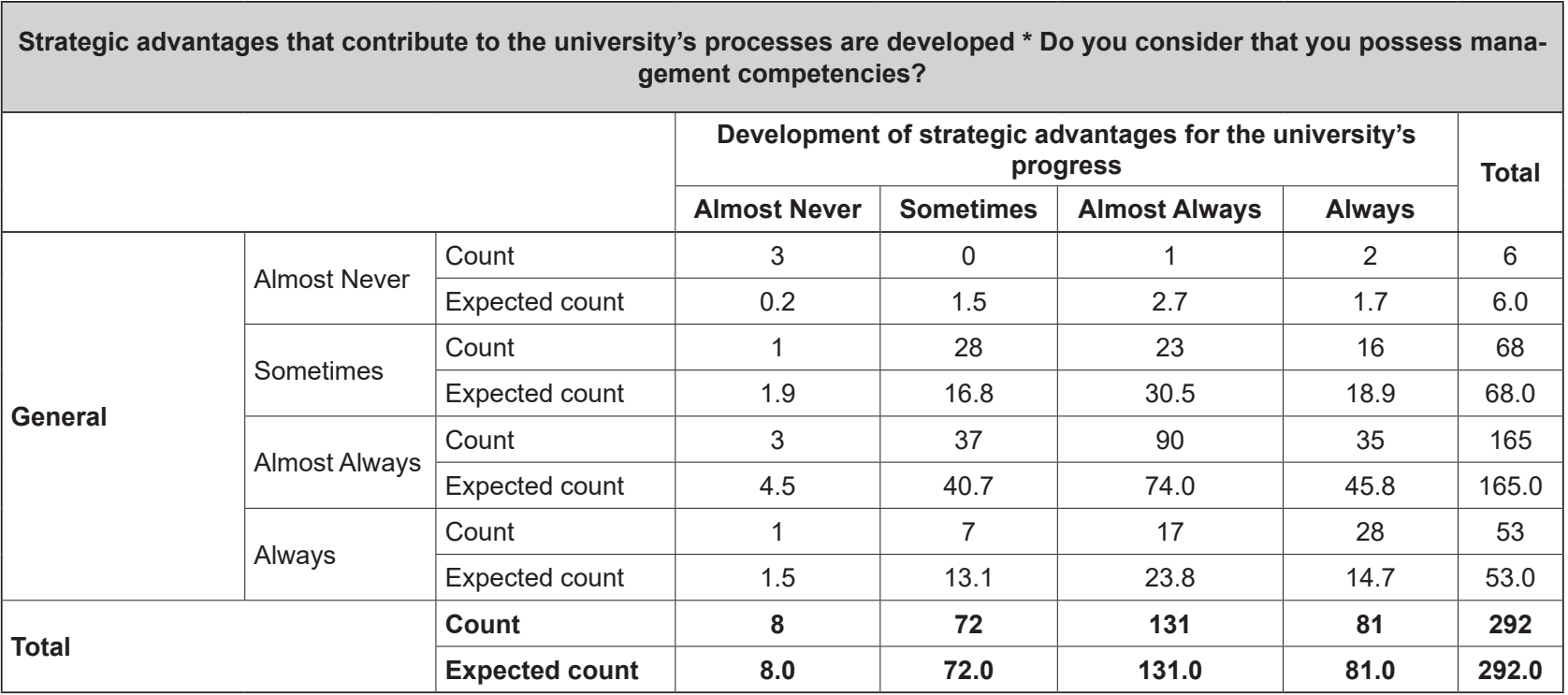

Source: Prepared by the author.

Table 7. Chi-Square Test - General Hypothesis.

\begin{tabular}{|l|c|c|c|}
\hline \multicolumn{4}{|c|}{ Chi-Square Tests } \\
\hline & Value & df & Asymptotic Significance (2-tailed) \\
\hline Pearson Chi-Square & $85.478^{\text {a }}$ & 9 & 0.000 \\
\hline Likelihood Ratio & 47482 & 9 & 0.000 \\
\hline Linear-by-Linear Association & 17990 & 1 & 0.000 \\
\hline N of Valid Cases & $\mathbf{2 9 2}$ & & \\
\hline
\end{tabular}

Source: Prepared by the author.

a. 7 cells $(43.8 \%)$ have expected count less than 5 . The minimum expected count is 0.16 .

Table 8. Data Cross Checking - Specific Hypothesis 1.

\begin{tabular}{|c|c|c|c|c|c|c|c|}
\hline \multicolumn{8}{|c|}{$\begin{array}{c}\text { Do you consider that you possess the skills to face the frequent changes in your work environment? * Strategic advantages } \\
\text { that contribute to the university's processes are developed }\end{array}$} \\
\hline & & & & Level of & training & & Ta \\
\hline & & & Almost Never & Sometimes & Almost Always & Always & Total \\
\hline \multirow{10}{*}{$\begin{array}{l}\text { Strategic advantage pro- } \\
\text { posal for the university's } \\
\text { progress }\end{array}$} & \multirow{2}{*}{ Never } & Count & 0 & 0 & 1 & 0 & 1 \\
\hline & & Expected count & 0.0 & 0.2 & 0.6 & 0.2 & 1.0 \\
\hline & \multirow{2}{*}{ Almost Never } & Count & 0 & 4 & 2 & 0 & 6 \\
\hline & & Expected count & 0.1 & 1.4 & 3.4 & 1.1 & 6.0 \\
\hline & \multirow{2}{*}{ Sometimes } & Count & 4 & 12 & 27 & 2 & 45 \\
\hline & & Expected count & 0.9 & 10.6 & 25.3 & 8.2 & 45.0 \\
\hline & \multirow{2}{*}{ Almost Always } & Count & 1 & 37 & 102 & 26 & 166 \\
\hline & & Expected count & 3.4 & 39.2 & 93.2 & 30.1 & 166.0 \\
\hline & \multirow{2}{*}{ Always } & Count & 1 & 16 & 32 & 25 & 74 \\
\hline & & Expected count & 1.5 & 17.5 & 41.6 & 13.4 & 74.0 \\
\hline \multirow{2}{*}{\multicolumn{2}{|c|}{ Total }} & Count & 6 & 69 & 164 & 53 & 292 \\
\hline & & Expected count & 6.0 & 69.0 & 164.0 & 53.0 & 292.0 \\
\hline
\end{tabular}

Source: Prepared by the author. 
Data presented in Tables 10 and 11 support the hypothesis.

The significance value is $0.002<0.05$, therefore, the null hypothesis $\mathrm{H}_{0}$ is rejected and $\mathrm{H}_{1}$ is accepted.

\section{DISCUSSION}

Following the analysis of the information collected, it was found that competency-based management and organizational development are related. Some deficiencies and problems that require a solution were also found among the administrative employees at the university's headquarters; it is therefore necessary to introduce a competency-based management model in the area of human resources management so that people in administrative positions may improve their performance. The selection of human talent is an action aimed at finding the

Table 9. Chi-Square Test - Specific Hypothesis1.

\begin{tabular}{|l|c|c|c|}
\hline \multicolumn{4}{|c|}{ Chi-Square Tests } \\
\hline & Value & Df & Asymptotic Significance (2-tailed) \\
\hline Pearson Chi-Square & $38.121^{\mathrm{a}}$ & 12 & 0.000 \\
\hline Likelihood Ratio & 34463.000 & 12 & 0.001 \\
\hline Linear-by-Linear Association & 15825.000 & 1 & 0.000 \\
\hline N of Valid Cases & 292 & & \\
\hline
\end{tabular}

Source: Prepared by the author.

a. 11 cells $(55.0 \%)$ have expected count less than 5 . The minimum expected count is 0.02 .

Table 10. Cross Reference - Specific Hypothesis 2.

\begin{tabular}{|c|c|c|c|c|c|c|c|}
\hline \multicolumn{8}{|c|}{$\begin{array}{c}\text { Practices leadership, manages change, and interacts with the staff * Proposes strategic advantages that contribute to the } \\
\text { university's processes }\end{array}$} \\
\hline & & & \multicolumn{4}{|c|}{ Leadership and Change Management } & \multirow{2}{*}{ Total } \\
\hline & & & Almost Never & Sometimes & Almost Always & Always & \\
\hline \multirow{10}{*}{$\begin{array}{l}\text { Strategic advantage pro- } \\
\text { posal for the university's } \\
\text { progress }\end{array}$} & \multirow{2}{*}{ Never } & Count & 0 & 1 & 4 & 0 & 5 \\
\hline & & Expected count & 0.0 & 0.11 & 2.9 & 0.9 & 5.0 \\
\hline & \multirow{2}{*}{$\begin{array}{l}\text { Almost } \\
\text { Never }\end{array}$} & Count & 1 & 2 & 2 & 0 & 5 \\
\hline & & Expected count & 0.1 & 1.2 & 2.8 & 0.9 & 5.0 \\
\hline & \multirow{2}{*}{ Sometimes } & Count & 1 & 23 & 35 & 3 & 62 \\
\hline & & Expected count & 1.3 & 14.4 & 35.0 & 11.4 & 62.0 \\
\hline & \multirow{2}{*}{$\begin{array}{l}\text { Almost } \\
\text { Always }\end{array}$} & Count & 2 & 30 & 80 & 27 & 139 \\
\hline & & Expected count & 2.9 & 32.2 & 78.4 & 25.5 & 139.0 \\
\hline & \multirow{2}{*}{ Always } & Count & 2 & 11 & 42 & 23 & 78 \\
\hline & & Expected count & 1.6 & 18.1 & 44.0 & 14.3 & 78.0 \\
\hline \multirow{2}{*}{\multicolumn{2}{|c|}{ Total }} & Count & 6 & 67 & 163 & 53 & 289 \\
\hline & & Expected count & 6.0 & 67.0 & 163.0 & 53.0 & 289.0 \\
\hline
\end{tabular}

Source: Prepared by the author.

Table 11. Chi-Square Test - Specific Hypothesis 2.

\begin{tabular}{|l|c|c|c|}
\hline \multicolumn{4}{|c|}{ Chi-Square Tests } \\
\hline & Value & Df & Asymptotic Significance (2-tailed) \\
\hline Pearson Chi-Square & $36.098^{\text {a }}$ & 15 & 0.002 \\
\hline Likelihood Ratio & 34473.000 & 15 & 0.003 \\
\hline Linear-by-Linear Association & 13877.000 & 1 & 0.000 \\
\hline N of Valid Cases & 289 & & \\
\hline
\end{tabular}

Source: Prepared by the author.

a. 15 cells $(62.5 \%)$ have expected count less than 5 . The minimum expected count is 0.02 . 
person who possesses the competencies, knowledge and other elements, such as academic degrees, necessary to obtain a specific job position within an organization.

As for the selection process used by the university, certain instruments were designed to efficiently supply suitable personnel. In addition, each department's management team attempts to establish stimulation and alignment processes in order to constantly improve the performance of its functions. In addressing these needs, the administrative staff attempts to maintain organizational development within the central administration.

It is essential that the human resources general office expedite the process of implementing the SERVIR guidelines for the application of the Position Profile Manual by management competencies in administrative positions. Also, considering each of the indicators assessed in this study, it can be stated that there are some situations to which more attention should be paid in order to raise the observations, despite the fact that the activities determined by position are being carried out.

A competency-based job profile design proposal was developed from the data analysis to contribute to the general objective (see Appendix 1). It sets out the occupations and competencies necessary for the adequate development of functions. It also contributes to the improvement of SERVIR forms at the university via the inclusion of fundamental competencies, the improvement of the level of development for the performance of functions, the adjustment of personnel to the job position, the introduction of a dictionary of managerial competencies and the adequate description of the job profile.

The use of a competency-based management guide within universities would benefit organizational development as stated by Rodríguez (2015):

Mejora la precisión para determinar el nivel de ajuste y potencial de una persona para diferentes trabajos, de tal forma que los planes de desarrollo puedan relacionarse mejor con las áreas donde realmente se necesita, tal como se puede corroborar en los estudios que sobre la materia ha desarrollado Alles (2009), en los que da cuenta de la importancia de los programas de desarrollo de las personas bajo el modelo de competencias y su relevancia para el crecimiento y continuidad de las organizaciones. [It improves the accuracy to determine a person's potential and level of adjustment to different jobs, so that development plans may be better related to the areas where they are really needed, as can be verified in the studies conducted by Alles (2009), in which the importance of people development programs following the competency model and its relevance for the growth and continuity of organizations is explained.] (p. 358)

Performance evaluation processes for administrative positions must also be considered in order to monitor employees' development and use that information to develop training programs. For organizations to remain at the forefront, they must update their processes and adopt new approaches that favor the development of employees' functions, in order to optimize resources and foster proper organizational development.

\section{CONCLUSIONS}

- Competency-based management and organizational development are correlated; therefore, the role of the human resources department at the university is essential to recruit the best talent for administrative positions, based on competency-based management that influences organizational development.

- Low levels of support regarding the needs of personnel are observed. Evidence of this can be found in the item of perceived support for employees' development within the university, where $32.6 \%$ stated that no adequate support was provided to perform their functions satisfactorily.

- No specific measures have been established by the university regarding personnel selection formats that include the concept of competencies for each job profile.

\section{RECOMMENDATIONS}

- It is essential to devise tools to measure the competencies established during the development of the tasks and thus be able to implement the processes required to enhance management competencies. Greater competitiveness should be pursued in the fulfillment of objectives and the institutional mission in order to achieve academic success.

- A document describing and specifying the essential functions and responsibilities of 
the position profile, including a competency assessment, should be prepared.

- Training budgets should be used to prepare the management team members for the efficient and effective performance of their functions. Furthermore, their performance should be assessed in a collaborative fashion.

\section{REFERENCES}

[1] Alles, M. (2008). Dirección Estratégica de Recursos Humanos Gestión por Competencias. Buenos Aires, Argentina: Ediciones Granica S.A.

[2] Alles, M. (2009). Diccionario de competencias: La trilogía. Las 60 competencias más utilizadas. Buenos Aires, Argentina: Ediciones Granica S.A.

[3] Becker, G. (1964). Human Capital. New York, United States: Columbia University.

[4] Chiavenato, I. (2002). Gestión del Talento Humano ( $2^{\text {nd }}$ ed.). Santafé de Bogotá, Colombia: McGraw Hill.

[5] Chiavenato, I. (2006). Introducción a la Teoría General de la Administración ( $7^{\text {th }}$ ed.). México D.F., Mexico: McGraw Hill.

[6] De la Cruz, G. (1999). Problemas y principios para el desarrollo de las organizaciones modernas. Gestión en el Tercer Milenio, 2(3), 45-54.

[7] Delgado, L. (2002). La Gestión Universitaria. In C. Aljovín, \& C. Germaná (Eds.), La Universidad en el Perú (pp. 81-88). Lima, Peru: Fondo Editorial UNMSM.

[8] Directiva No. 001-2016-SERVIR/GDSRH. Normas para la Gestión del Proceso de Diseño de Puestos y Formulación del Manual de Perfiles de Puesto - MPP. Diario Oficial El Peruano (2016).
[9] Espinoza, N. (2000). Gerencia Universitaria: Universidad peruana y tercer milenio. Lima, Peru: Editorial San Marcos.

[10] Fernández, L. (2008). Análisis de la gestión universitaria en la universidad pública peruana [paper]. VIII Coloquio Internacional sobre Gestión Universitaria en América del Sur, Asunción, Paraguay.

[11] Fernández, J. (2006). Gestión por competencias. Madrid, Spain: Pearson Educación S.A.

[12] Hernández, R., Fernández C., \& Baptista P. (2010). Metodología de la Investigación. Bogotá, Colombia: McGraw Hill.

[13] McClelland, D. (1973). Testing for Competence Rather Than for "Intelligence". American Psychologist, 28(1), 1-14.

[14] Resolución Rectoral No. 01206-R-11. Reglamento de Organizaciones y Funciones de la Universidad Nacional Mayor de San Marcos (2011).

[15] Rodríguez, J. (2015). Implementación del modelo de gestión de talento humano por competencias en una universidad de Lima Metropolitana. Propósitos y Representaciones, 3(2), 319-401

[16] Sánchez, G. (2009). El Desarrollo Organizacional: Una Estrategia de Cambio para las Instituciones Documentales. Anales de Documentación, (12),235-254. ISSN: 15752437.

[17] Spencer L., \& Spencer S. (1993). Competence at work: Models for Superior Performance. New York, United States: Wiley \& Sons. 


\section{APPENDIX}

Appendix 1. Proposed competency-based profile.

\begin{tabular}{|l|l|}
\hline SPECIFIC MANAGEMENT COMPETENCIES & $\begin{array}{l}\text { Ability to manage administrative staff. Ability to generate and nurture the } \\
\text { talent within their staff and provide feedback. }\end{array}$ \\
\hline Empowerment & $\begin{array}{l}\text { Ability to work with his/her administrative staff as a team. Ability to devise } \\
\text { and pursue performance objectives focused on improving and strengthe- } \\
\text { ning the talent of their personnel. Ability to obtain good results through the } \\
\text { integration of their administrative staff, leveraging their diversity and assig- } \\
\text { ning value to the administrative processes. }\end{array}$ \\
\hline Executive Leadership & $\begin{array}{l}\text { Ability to develop and work with the leaders of other departments with the } \\
\text { aim of creating a better organizational climate and commitment. }\end{array}$ \\
\hline Strategic Vision & $\begin{array}{l}\text { Ability to foresee and understand changes. Ability to suggest ways to impro- } \\
\text { ve the administrative environment. }\end{array}$ \\
\hline Leading by Example & $\begin{array}{l}\text { Ability to convey strategies and values through a prototype of administrative } \\
\text { worker management. Ability to promote the generation of ideas in a suppor- } \\
\text { tive environment. }\end{array}$ \\
\hline
\end{tabular}

\section{SPECIFIC COMPETENCES BY DEPARTMENT}

\section{FUNCTIONS}

1. Plan, manage, regularize, inspect and review administrative systems and methods used within the university environment.

2. Manage financial administrative processes, supply procedures and other services in their area of competence.

3. Manage and oversee the university public investment projects (PIP) and the procurement of goods.

4. Establish special commissions according to the matters of interest via Directorial Resolutions (DR).

5. Approve the administrative bases via the RD in force.

6. Formulate technical opinions in administrative and financial terms on university administration.

7. Propose policies for the improvement of the institutional administrative systems.

8. Guide the high authority of the university in matters related to the administrative and financial systems it administers.

9. Work together with the regulatory entities of the administrative-financial regimes of public administration

10. Plan and carry out training on the application of management systems, finances, among others, pertaining to university administration.

11. Other tasks established by the high authority.

\begin{tabular}{|c|c|c|c|}
\hline \multirow{2}{*}{ COMPETENCIES } & \multicolumn{3}{|c|}{ STRUCTURE } \\
\hline & Knowledge & Skills & Attitudes \\
\hline \multirow{4}{*}{$\begin{array}{l}\text { Planning and } \\
\text { organization }\end{array}$} & $\begin{array}{l}\text { Administrative } \\
\text { control }\end{array}$ & $\begin{array}{l}\text { Team manage- } \\
\text { ment }\end{array}$ & $\begin{array}{l}\text { Achievement and } \\
\text { results orientation }\end{array}$ \\
\hline & $\begin{array}{l}\text { Financial mana- } \\
\text { gement }\end{array}$ & $\begin{array}{l}\text { Control of finan- } \\
\text { cial resources }\end{array}$ & $\begin{array}{l}\text { Achievement and } \\
\text { results orientation }\end{array}$ \\
\hline & $\begin{array}{l}\text { Development of } \\
\text { operational plans }\end{array}$ & $\begin{array}{l}\text { Budget mana- } \\
\text { gement }\end{array}$ & $\begin{array}{l}\text { Achievement and } \\
\text { results orientation }\end{array}$ \\
\hline & $\begin{array}{l}\text { Administrative } \\
\text { file management }\end{array}$ & $\begin{array}{l}\text { Team manage- } \\
\text { ment }\end{array}$ & $\begin{array}{l}\text { Achievement and } \\
\text { results orientation }\end{array}$ \\
\hline \multirow{2}{*}{ Decision-making } & $\begin{array}{l}\text { Development } \\
\text { of technical-ad- } \\
\text { ministrative } \\
\text { provisions }\end{array}$ & $\begin{array}{l}\text { Public process } \\
\text { management }\end{array}$ & Negotiation power \\
\hline & $\begin{array}{l}\text { University mana- } \\
\text { gement }\end{array}$ & $\begin{array}{l}\text { University } \\
\text { resource mana- } \\
\text { gement }\end{array}$ & Problem solving \\
\hline \multirow{2}{*}{$\begin{array}{l}\text { Management } \\
\text { and achieve- } \\
\text { ment of objec- } \\
\text { tives }\end{array}$} & $\begin{array}{l}\text { Development } \\
\text { of technical-ad- } \\
\text { ministrative } \\
\text { provisions }\end{array}$ & $\begin{array}{l}\text { Administration } \\
\text { and control }\end{array}$ & $\begin{array}{l}\text { Achievement and } \\
\text { results orientation }\end{array}$ \\
\hline & $\begin{array}{l}\text { Management of } \\
\text { administrative } \\
\text { resources }\end{array}$ & $\begin{array}{l}\text { Administrative } \\
\text { capacity }\end{array}$ & $\begin{array}{l}\text { Management } \\
\text { responsibility }\end{array}$ \\
\hline $\begin{array}{l}\text { Planning and } \\
\text { organization }\end{array}$ & $\begin{array}{l}\text { Public manage- } \\
\text { ment }\end{array}$ & $\begin{array}{l}\text { Administration } \\
\text { and control }\end{array}$ & $\begin{array}{l}\text { Management } \\
\text { responsibility }\end{array}$ \\
\hline $\begin{array}{l}\text { Planning and } \\
\text { organization }\end{array}$ & $\begin{array}{l}\text { Human resour- } \\
\text { ces manage- } \\
\text { ment }\end{array}$ & $\begin{array}{l}\text { Communication } \\
\text { with administra- } \\
\text { tive staff }\end{array}$ & $\begin{array}{l}\text { Achievement and } \\
\text { results orientation }\end{array}$ \\
\hline $\begin{array}{l}\text { Working under } \\
\text { pressure }\end{array}$ & & & \\
\hline
\end{tabular}

Note: The positions of the General Directorate of Administration set forth in the ROF UNMSM were used as a basis.

Source: Prepared by the author. 